\title{
INVESTIGATIONS OF MECHANISMS OF MICROBIAL ENHANCED OIL RECOVERY BY MICROBES AND THEIR METABOLIC PRODUCTS
}

Topical Report
By
K. L. Chase
R. S. Bryant
K. M. Bertus
A. K. Stepp

December 1990

Work Performed Under Choperative Agreement No. DE-FC22-83FE60149

\author{
Prepared for \\ U.S. Department of Energy \\ Assistant Secretary for Fossil Energy
}
Edith Allison, Project Manager
Bartlesville Project Office
P.O. Box 1398
Bartlesville, OK 74005

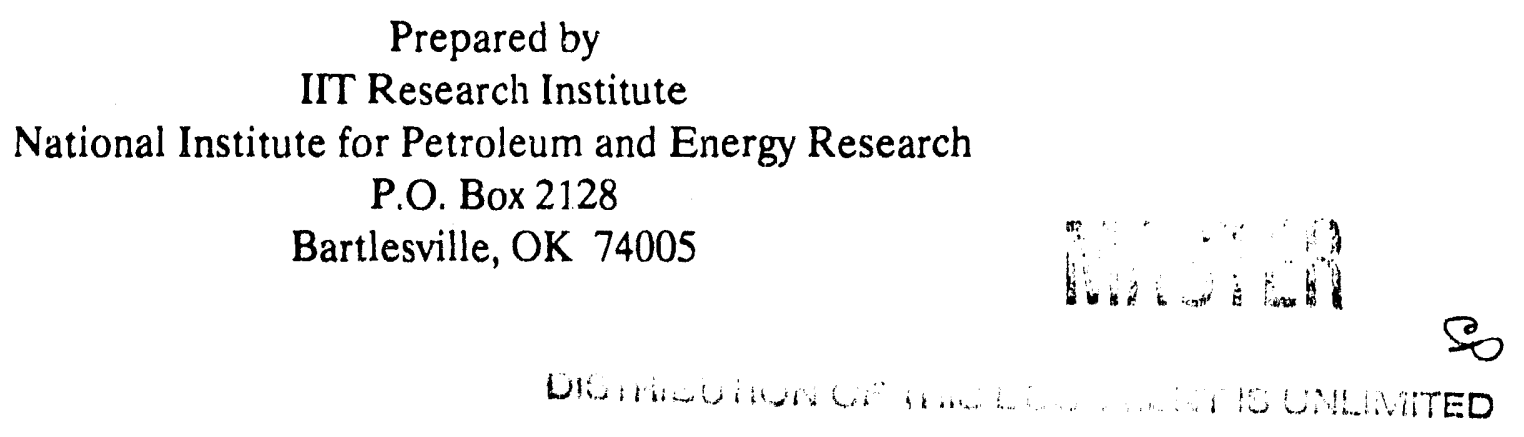



7. IFT determinations between chemicals representing NIPER 1 and 6 metabolic products and nonane

8. Interfacial tension determinations using varying salt concentrations ..............................18

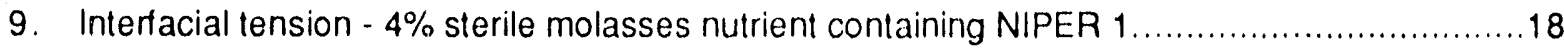

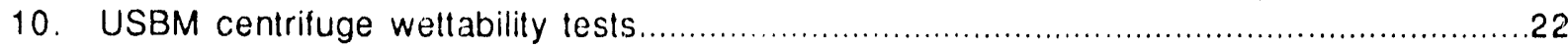

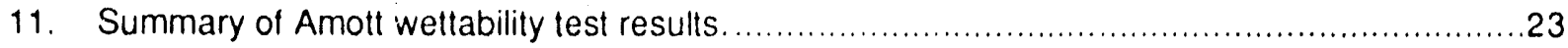

12. Summary of unsteady-state relative permeability tests...........................................24

Eiqure

\section{ILLUSTRATIONS}

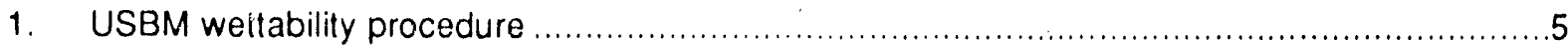

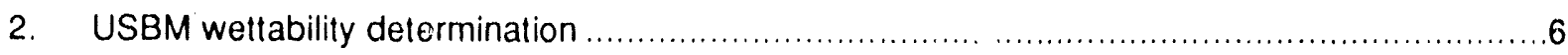

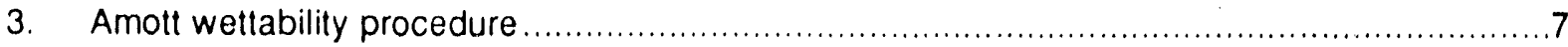

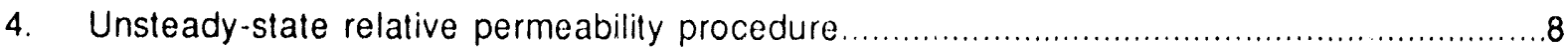

5. Anaerobic microbial counts of NIPER 1 and 6 in molasses and TSB ............................ 10

6. Metabolites detectable by gas chromatography from NIPER 1 and 6 growing in molasses and TSB

7. Microbial counts of NIPER 1 and 6 grown with and without sodium bicarbonate

8. Total metabolites detected by gas chromatography of NIPER 1 and 6 grown with and without sodium bicarbonate and/or Berea sandstone......

9. Total metabolites detected by gas chromatography of NIPER 1 grown in Medium $E$ and sterile molasses.....

10. Microbial counts of NIPER 1 and 6 in modified Medium E ............................................ 16

11. Residual crude oil after waterflooding micromodel ................................................. 19

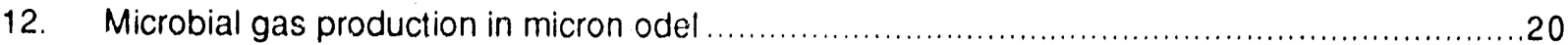

13. Microbial gas production and oil mobilization in micromodel.........................................20

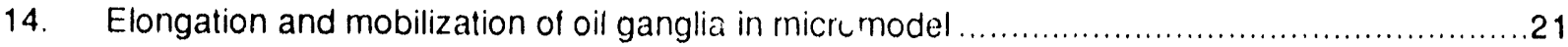

15. Crude oil solubilization after microbial treatment in micromodel .....................................21

16. Unsteady-state relative permeability fraction curves for cores with different permeability values

17. Unsteady-state relative permeability ratio curves for cores with different permeability values 


\title{
INVESTIGATION OF MECHANISMS OF MICROBIAL ENHANCED OIL RECOVERY BY MICROBES AND THEIR METABOLIC PRODUCTS
}

\author{
by K. L. Chase, R. S. Bryant, K. M. Bertus, and A. K. Stepp
}

\begin{abstract}
Priur work at NIPER has identified the mechanisms of oil mobilization by certain microbial formulations. Mechanisms that have been shown to be important include wettability alteration, emulsification, solubilization, and alteration in interfacial forces. Experiments at NIPER have demonstrated that oil mobilization by microbial formulations is not merely the result of the effects of the metabolic products from the in situ fermentation of nutrient. Further investigation into the interfacial properties of microorganisms and the concentrations of metabolic products at the oil-water interface is needed to determine the specific mechanisms of oil mobilization.

A combination of two microorganisms, Bacillus licheniformis, NIPER 1 (ATCC. No. 3930\%), and a Clostridium species (NIPER 6), was determined to be an effective microbial formulation for the recovery of residual crude oil in porous media. Flask tests with various nutrients and environmental conditions were used to evaluate the growth and metabolite production of NIPER 1 and 6 . Several interfacial tension (IFT) measurements were conducted using certain metabolic products from the combined microbial cultures NIPER 1 and NIPER 6. Nonane was used as the oil for these experiments, since crude oil from DelawareChilders field emulsifies very easily. Only propionic acid in the nonane system gave an IFT in the range of $1 \mathrm{mN} / \mathrm{m}$ by the spinning drop method.

The interfacial tensions of a selected microbial formulation were measured with two different crude oils using brines of varying salinities. Comparisons were made with saline brines containing only the nutrient and with microbial metabolite solutions from which the active cells have been removed by filtration to isolate the specific effects of the microbial cells. A significant change from the controls was not apparent in interfacial tensions using varying salinities or filtered products. However, a correlation between salinity and surface tension was observed; an increase in surface tension was observed with increased salinity.
\end{abstract}

Etched-glass micromodel studies showed that the microbial formulation effectively mobilized crude oil trapped after waterlooding. The observations from the micromodel indicate that significant interfacial effects occurred. Some oil mobilization by gas displacement was observed in the pores.

Because the interfacial tension values were not in the range $\left(10^{-2}\right.$ to $\left.10^{-3} \mathrm{mN} / \mathrm{m}\right)$ necessary to cause a sufficient increase in capillary number, other mechanisms including wettability alteration were also investigated. Certain microbial formulations appear to shift the wettability of Berea sandstone core samples toward a more water-wet condition. The microbial cells appear to be involved in this wettability alteration since no change was observed in samples tested with a microbial formulation that had been filtered to remove cells 
Unsteady-state relative permeability tests were performed with microbial formulations in Berea sandstone cores. A decrease in relative permeability to water and an increase in relative permeability to oil was usually observed in microbially flooded cores causing an apparent curve shift toward a more water-wet condition. Samples tested with the microbial formulation exhibited higher oil recovery before water breakthrough and lower residual oil saturations.

\section{INTRODUCTION}

Laboratory and field experiments have demonstrated the potential for mobilization of oil with microbes and microbial products, but the actual mechanisms involved in the process have not been fully identified. Mechanisms shown to be important in most enllanced oil recovery methods involve lowering the mobility ratio and/or increasing capillary number. A significant increase in capillary number is achieved by lowering the interfacial tension beiween the displacing and displaced fluids. It has been reported that a 100 to 1,000 -fold decrease in IFT is required to cause a decrease in oil saturation. ${ }^{1}$

Prior work has indicated that certain microbial formulations exhibit optimal behavior at specific salinities similar to surfactant systems. ${ }^{2}$ Interfacial tensinns of selected microbial formulations with crude oils were measured to determine the effects of salinity and composition of the microbial formulation.

Another significant mechanism of microbial oil mobilization is weftability alteration. The displacement of a wetting fluid by a nonwetting one is less efficient than the displacement of a nonwetting by a wetting fluid. For a microbial flooding process, wellability alteration could be a result of adsorption of a metabolic product or microbial cells. Tests were performed to determine wettability using the Amott ${ }^{3}$ imbibition and USBM ${ }^{4}$ centrifuge methods. Unsteady-state relative permeability tests were also performed to observe wettability alteration effects on relative permeability and oil recovery.

\section{MATERIALS}

\section{Microorganisms}

Results from previous studies have shown that a combination of Bacillus licheniformis, NIPER 1 (ATCC No. 39307), and a Clostridium species (NIPER 6) is one of the most effective formulations for the recovery of residual oil. This microbial formulation was chosen for further interfacial tension and wettability studies. Bacillus licheniformis is a facultatively anaerobic, sporeforming rod thal produces alcohols, biosurfactant, and some organic acids when fermenting molasses. The anaerobic sporeforming Clostridium is a member of the butyric acid group that produce acetone, butanol, ethanol, isopropanol, hutyric acid, acetic acid, propionic acid, carbon dioxide, and hydrogen when fermenting sucrose. This particular Clostridium species also produces a biosurfactant.

Several combinations of microorganisms were used in the USBM weltability tests. NIPER Bac 1 is the combination of four microorganisms used in a microbially enhanced waterlood field project in the Mink Unit of Delaware-Childers field near Nowata, Oklahoma. 5 NIPER BaC 1 is a combination of NIPER 1, NIPER 
2. NIPER 3, and NIPER 4. N.PER 2 is a species of Bacillus that produces surfactant, acids, and some carbon dioxide. NIPER 3 is a species of Clostridium similar to NIPER 6 that produces carbon dioxide, ethanol, butyric acid, and surfactant. NIPER 4 is a Gram (-) negative, facultatively anaerobic rod that produces carbon dioxide and acids when fermenting sucrose.

NIPER 1 alone was used for relative permeability and Amott wottability tests because USBM centrifuge wettability tests have shown NIPER 1 to be the most effective in alteration of the wettability index. NIPER 6 was eliminated from these tests because the abundant production of gas would interfere with the tests.

\section{Nutrient}

The molasses used in these experiments was obtained from Pacific Molasses Company in: Oklahoma City, and its mineral content is as follows: total ash, $6.1 \%$; calcium, $0.8 \%$; phosphorous, $0.08 \%$; magnesium, $0.35 \%$; potassium, $2.4 \%$; sulfur, $0.8 \%$; and sodium, $0.2 \%$. The amount of total suspended solids is $74 \%$, of which $3 \%$ is total protein, $48 \%$ is total sugar (sucrose), and the remaining $23 \%$ is fiber. The concentration of molasses used in the experiments was $4 \% \mathrm{vol} / \mathrm{vol}$ in tap water with $0.1 \% \mathrm{wt} / \mathrm{vol}$ ammonium phosphate added to facilitate microbial metabolism.

For relative permeability and Amott wettability tests, $3 \% \mathrm{wt} / \mathrm{vol}$ potassium chloride was added to the molasses solution. Microbes were cultivated in filtered molasses $(0.45 \mu$ Millipore filter) solution with an antibiotic added to prohibit growth of the normal molasses flora. Microbes were cultivated in tryptic soy broth (TSB) for the imbibition phase of the USBM wettability centrifuge tests.

A modified Medium E (Mod E) solution was used in some of the determinations of interfacial tension and for flask tests. The solution contained: sodium chloride, $5 \%$; glucose, $1 \%$; ammonium sulfate, $0.1 \%$; sodium nitrate, $0.1 \%$; magnesium sultate, $0.05 \%$; and yeast extract, $0.05 \%{ }^{6}$

\section{Crude Oil and Brine}

Delaware-Childers (DC) crude oil was obtained from the Bartlesville Sand formation in DelawareChilders field in northeastern Oklahoma. Delaware-Childers oil has a gravity of $31^{\circ} \mathrm{API}$ and a density of $0.87 \mathrm{~g} / \mathrm{cm}^{3}$. Chelsea-Alluwe $(C A)$ crude oil was obtained from the Bartlesville Sand formation of the Chelsea-Alluwe field. Chelsea-Alluwe oil has a density of 0.852 and a gravity of $34.6^{\circ} \mathrm{API}$. Brine with a concentration of $3 \%$ sodium chluride by weight was used for USBM wettability tests. Because of the possible presence of clays in the Berea sandstone cores, a concentration of $3 \% \mathrm{wt} / \mathrm{vol}$ potassium chloride was used for Amoll wettability and relative permeability tests. 


\section{Core Samples}

Blocks of Berea sandstone were obtained from Cleveland Quarries (Amherst, Ohio) and cut into cylindrical cores of 1.5 inches $(3.8 \mathrm{~cm})$ in diameter and 3 inches $(7.6 \mathrm{~cm})$ in length for Amott wettability and relative permeability tests. Core plugs for the USBM centrifuge tests were drilled $0.75 \mathrm{in} .(1.9 \mathrm{~cm})$ in diameter and 1.2 in. $(3 \mathrm{~cm})$ in length.

\section{METHODOLOGY}

\section{Elask Tests}

Flask tests were conducted in $500-\mathrm{mL}$ nephelometric flasks. Optical densities were measured at 530 nanometers. The standard microbial plate count method ${ }^{7}$ was used to enumerate microorganisms. Selective media developed and tested at NIPER were used for enumeration of NIPER 1 and NIPER 6.

\section{Surface and Interfacial Tension}

Surface tensions and some of the interfacial tensions were measured with a Fisher Instruments semi-automated ring tensiometer by the ASTM method ${ }^{8}$ using the DuNuoy principle. Interfacial tensions were measured with the spinning drop interfacial tensiometer by the method of Wade, et al. ${ }^{9}$

\section{Gas Chromatography}

Compositional analyses were performed using a Hewlett Packard 5980A gas chromatograph equipped with a flame ionization detector. A 6-ft $(1.83-\mathrm{m})$ glass column packed with Poropak QS $(800$ 100 mesh) was used for all analyses. A temperature program of $95^{\circ}$ to $195^{\circ} \mathrm{F}\left(35^{\circ}\right.$ to $\left.90.6^{\circ} \mathrm{C}\right)$ gave the best separation of compounds. Standards used were 0.1 or $1 \%$ by weight alcohols or fatty acids.

\section{Coreflood Apparatus}

Three-inch-long core samples for Amott wettability and relative permeability tests were encased in rubber sleeves and confined in a hydrostatic coreholder at $350 \mathrm{psi}$, net confining pressure. The samples were waterflooded at constant pressure using nitrogen to drive a piston-type fluid reservoir.

\section{Core Preparation}

All core samples were evacuated and then saturated with brine. Initial water saturation $\left(S_{w i}\right)$ was established in USBM centrifuge samples by placing the samples in Delaware-Childers crude oil and centrifuging at approximately 3,000 RPM which is equivalent to a pressure gradient of approximately 10 psi. One set of the centrifuge plugs was stored for 7 days submersed in the crude to establish initial wettability conditions. Other sets of core plugs were left in the oil overnight.

Samples were prepared for Amott wettability and relative permeability tests by flushing with a refined mineral oil (45 CP, viscosity). The mineral oil was then displaced with crude oil by flushing with brine. Permeability to oil at initial vater saturation was determined using Darcy's law. All core samples to be used for wettability and relative permeability tests were aged with the crude oil in place to establish initial wettability conditions. The samples were stored submersed irı the crude for 7 days. 


\section{Micromodel Experiments}

Glass micromodels used in this study were previously described by Chatzis ${ }^{10}$ and Bryant and Douglas. ${ }^{11}$ The flow rate was adjusted to $0.01 \mathrm{~mL} / \mathrm{min}$, which corresponded to approximately $8 \mathrm{tt} /$ day. The micromodels were brine-saturated $(0.5 \%$ sodium chloride), oil-saturated with crude oil from ChelseaAlluwe field, and waterflooded to residual oil saturation before microbial injection.

\section{Centrifuge USBM Wettability Tests}

For these tests, core samples were stored at initial water saturation and submersed in the crude oil for 7 days. Other samples were stored for 24 hours. Imbibition and drainage capillary pressure curves with brine and crude oil were generated by increasing rates of rotation to facilitate oil displacement with brine followed by brine displacement with oil. For other samples, imbibition and drainage capillary pressure curves were generated using a microbial formulation in TSB. The displacement curves of average saturation versus pressure were used to calculate the wettability index. A schematic of the procedure for USBM wettability is presented in figure 1 , and a schematic showing wettability determination is presented in figure 2.

\section{USSBMI WYEI'I'ABIILIIIY}

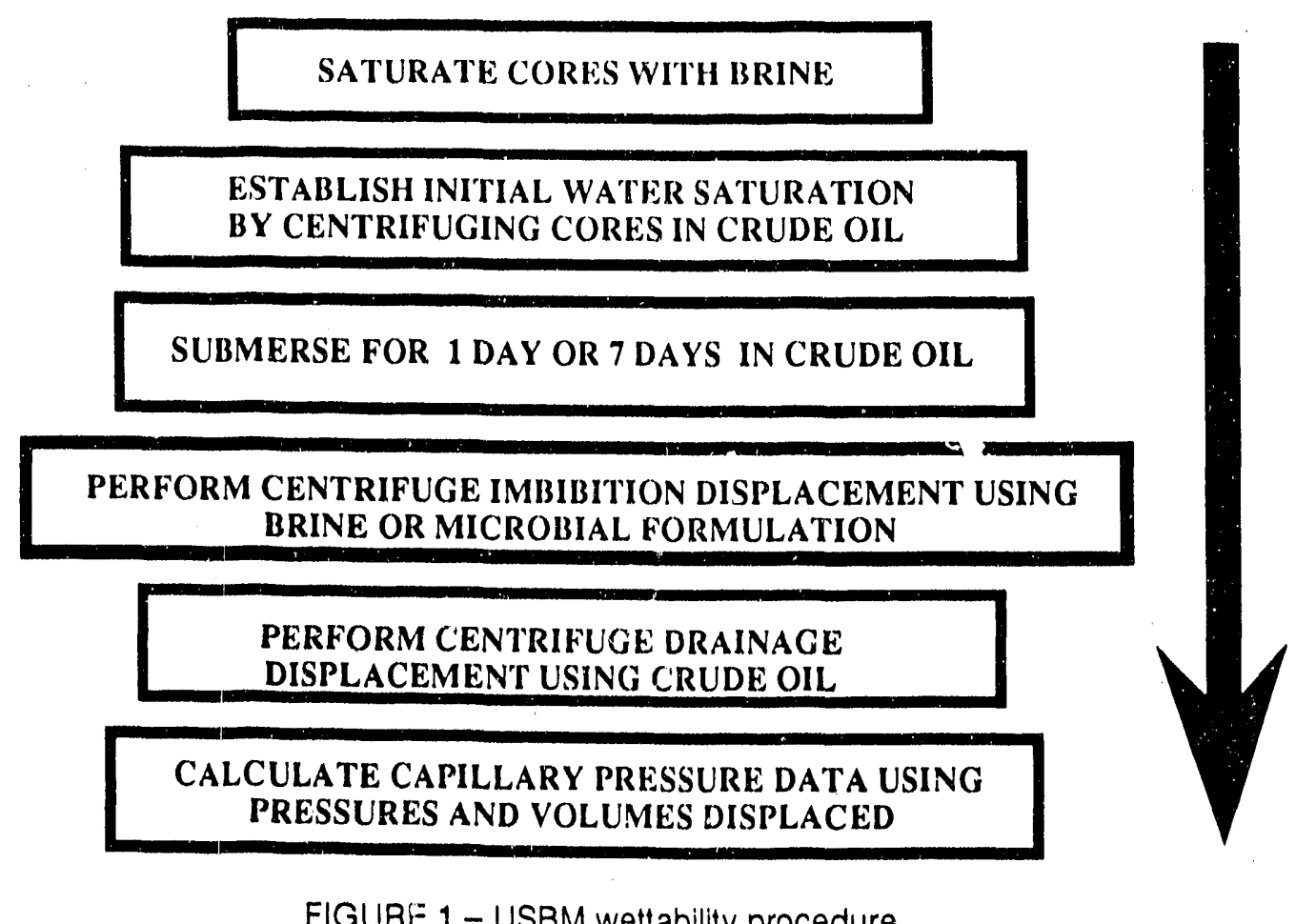

FIGURE 1-- USBM wettability procedure. 


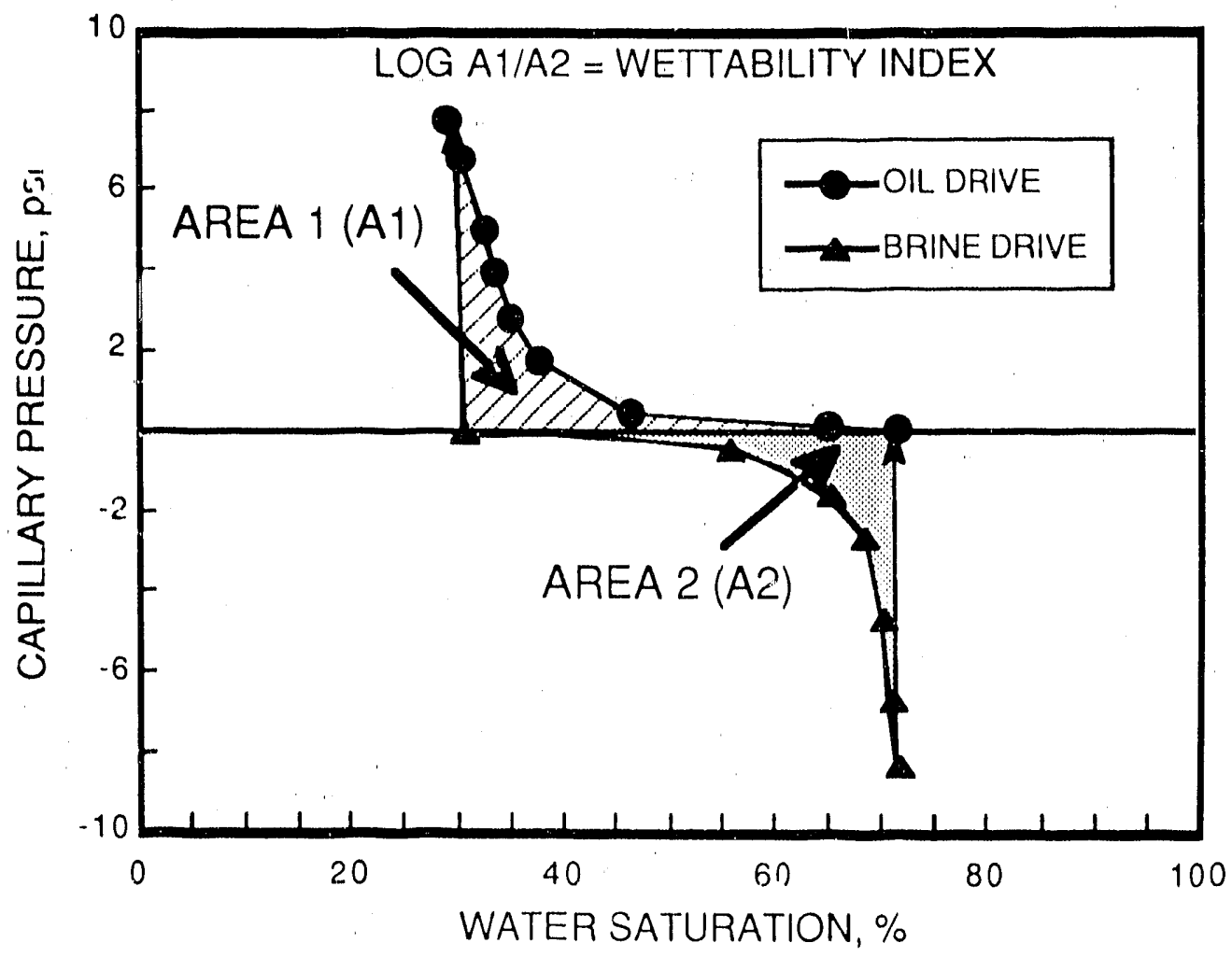

Figure 2.- USBM w'ellability determination.

\section{Amott Wettability Tests}

Following a 7-day period submersed in crude oil, the cores were placed in imbibition tubes. One core was placed in a tube full of brine solution (A1), and the other core was placed in a tube full of the microbial formulation (A4). The cores were left in the lubes and monitored daily until no more oil was produced. Both samples were stored in the tubes for about 3 weeks.

Each sample was then removed from the wettability tube and loaded into a coreholder. The samples were flooded with either brine or microbial formulation to determine the additional volume of mobil z oil that could be displaced dynamically. Sample A4 was flooded with the microbial formulation. The samples were then placed in imbibition tubes full of crude oil. Again, the samples were left in the tubes until equilibrium had been reached, then both samples were flushed with crude oil until no wore brine (or microbial formulation) was produced. Residual water saturation was verified by a toluene distillation method. Results are presented in terms of a wettability index to both oil and water. A brief outline of the Amott wettability procedure is presented in figure 3. 


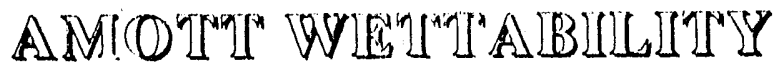

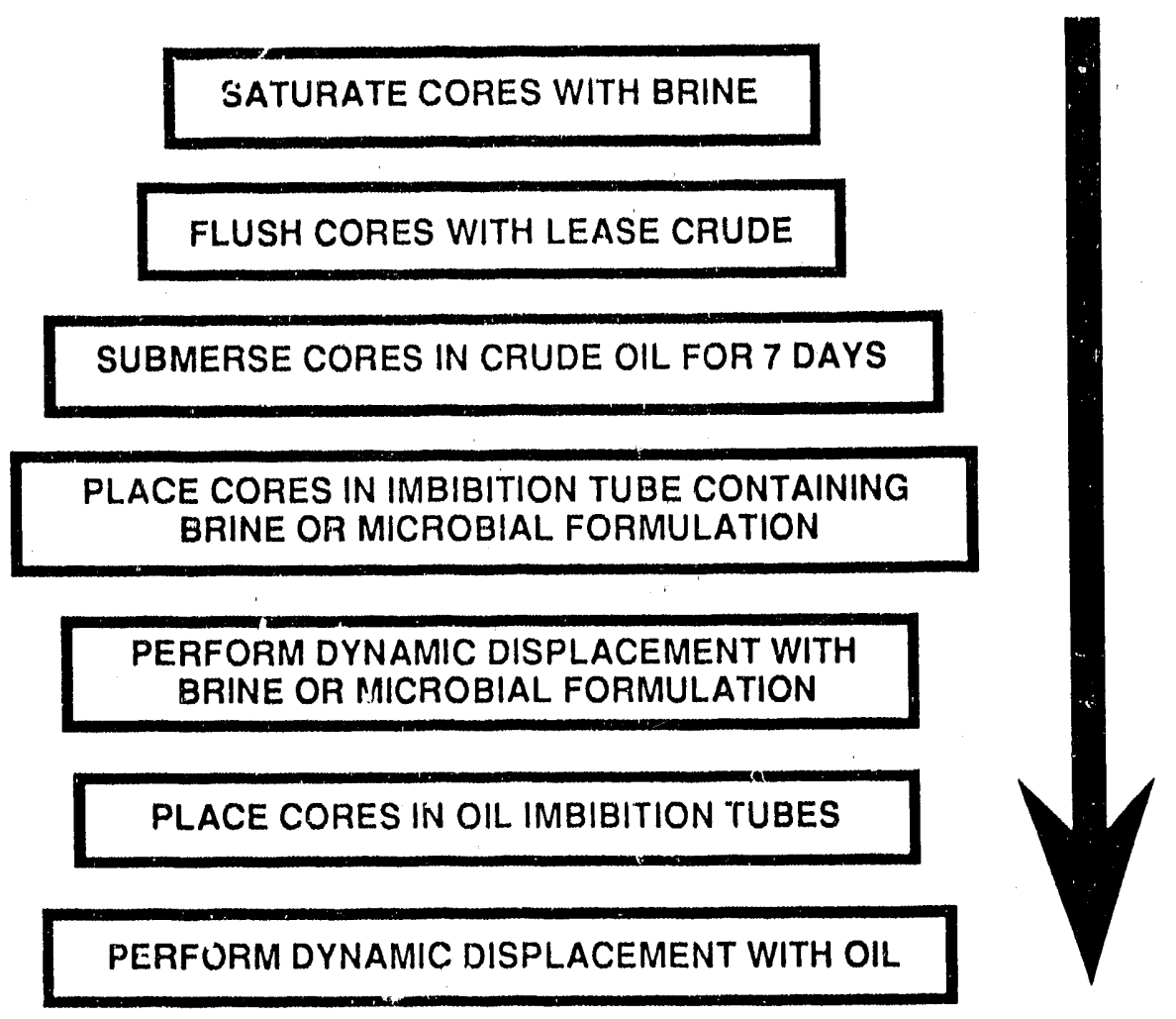

FIGURE 3.- Amott wettability procedure.

\section{Unsteady-State Relative Permeability Tests}

After the cores were aged in crude oil for 1 week, the crude oil was displaced dynamically with mineral oil. Permeability to mineral oil at initial water saturation was determined. The core was immediately waterflooded with either the $3 \%$ by weight potassium chloride brine or the microbial formulation. The cores were waterflooded at a constant pressure calculated from a theoretical flow rate of $0.1 \mathrm{~cm}^{3} / \mathrm{sec}$ to overcome end effects. The brine (or microbial formulation) was injected at constant pressure, and water and oil volumes produced were measured as a function of time. These data were used to calculate relative permeability values for water and oil using the method of Johnson, Bossler and Naumann. ${ }^{12}$ A schematic of the procedure for sample preparation and relative permeability is presented in figure 4 . 


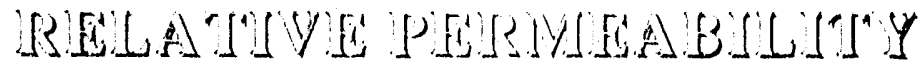

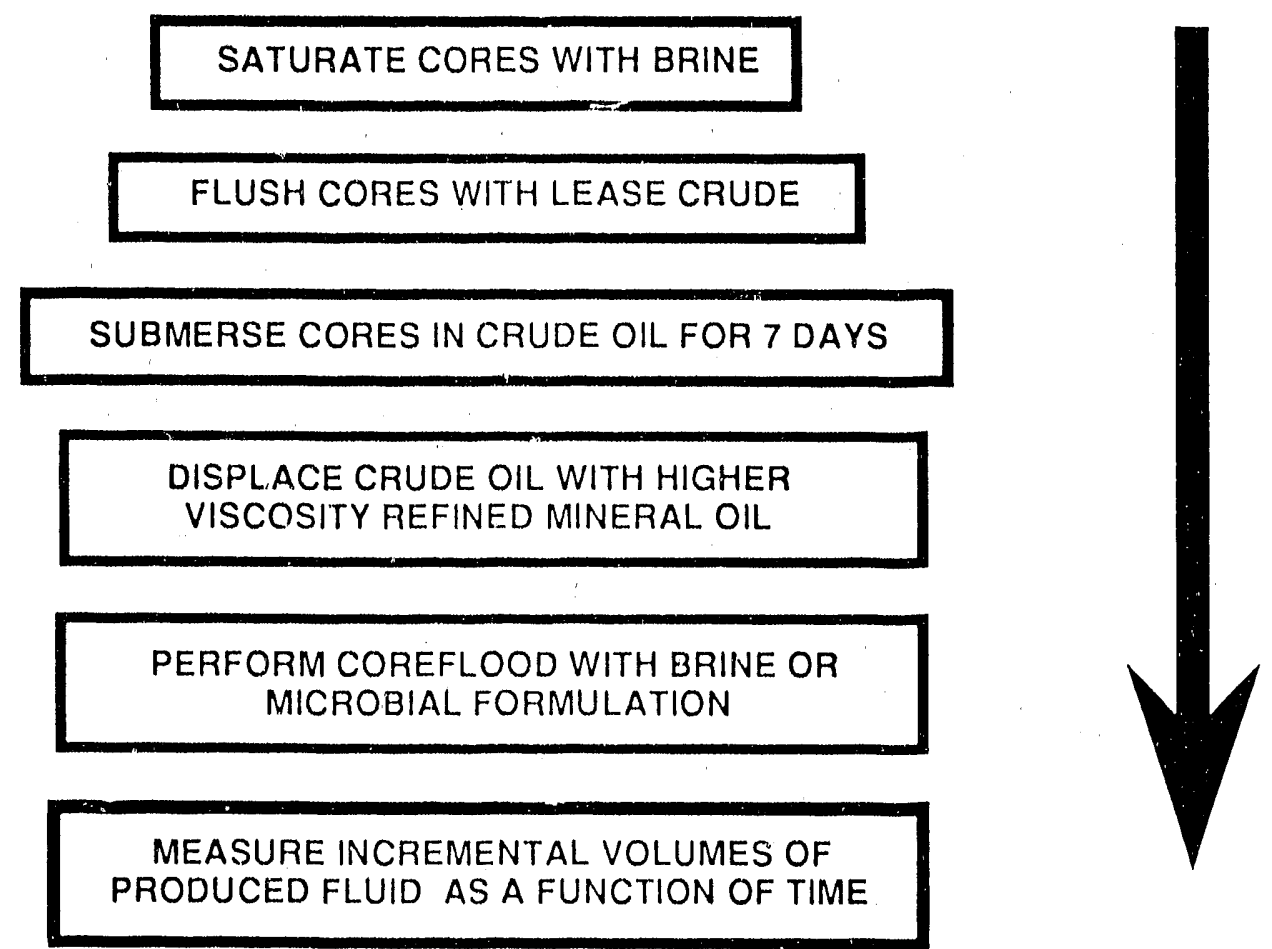

FIGURE 4.- Unsteady-state relative permeability procedure.

\section{RESULTS AND DISCUSSION}

\section{Flask Tests}

A series of flask tests was conducied with NIPER 1 and 6 using different nutrients and salt concentrations to determine the differences in microbial growlh and metabolite prodıction. One flask test compared TSB with molasses, another compared differences oblained with crushed Berea sandstone and $1 \%$ sodium bicarbonate in the flasks, and the last llask tust compared NIPER 1 and 6 microbial growth and metabolite production in modified Medium $E$ with the results in molasses.

The results from a comparison of NIPER 1 and 6 in TSB vS. molasses are presented in tables 1 and 2 and figures 5 and 6 . Although the microbial counts for boll NIPER 1 and NIPER 6 were consistently 10 times higher in TSB than molasses, the metabolites detected by gas chromatography were almost twice as high in the molasses flask. The metabolite responsible for most of this increase was butyric acid. However, NIPER 1 also appears to produce butyric acid, so it is unclear which microorganism was stimulated by the molasses. 
TABLE 1.- Microbial counts and metabolites detected by gas chromatography from NIPER 1 and 6 in molasses

\begin{tabular}{rlccc}
\hline Time, hr & $\begin{array}{c}\text { NIPER 1 Aerobic, } \\
\text { cfu/mL }\end{array}$ & $\begin{array}{c}\text { NIPER 1 Anaerobic, } \\
\text { cfu/mL }\end{array}$ & $\begin{array}{c}\text { NIPER 6, } \\
\text { cfu/mL }\end{array}$ & $\begin{array}{c}\text { TGC, } \\
\text { wt\% }\end{array}$ \\
\hline 20 & $1.2 \times 10^{6}$ & $9.8 \times 10^{5}$ & $1.6 \times 10^{6}$ & 0.25 \\
4 & $2.9 \times 10^{5}$ & $8.5 \times 10^{5}$ & $2.2 \times 10^{6}$ & 0.24 \\
8 & $2.8 \times 10^{7}$ & $6.2 \times 10^{6}$ & $2.8 \times 10^{7}$ & 0.24 \\
12 & $1.9 \times 10^{6}$ & $4.6 \times 10^{7}$ & $2.8 \times 10^{7}$ & 0.24 \\
24 & $8.0 \times 10^{7}$ & $5.6 \times 10^{7}$ & $4.0 \times 10^{5}$ & 0.24 \\
48 & $8.0 \times 10^{8}$ & $1.4 \times 10^{7}$ & $5.5 \times 10^{6}$ & 0.31 \\
72 & $5.0 \times 10^{7}$ & $1.3 \times 10^{8}$ & $1.2 \times 10^{7}$ & 0.43 \\
96 & $1.3 \times 10^{8}$ & $1.0 \times 10^{8}$ & $1.5 \times 10^{7}$ & 0.53 \\
120 & $1.1 \times 10^{7}$ & $3.1 \times 10^{8}$ & $1.7 \times 10^{6}$ & 0.57 \\
144 & $3.3 \times 10^{6}$ & $1.1 \times 10^{7}$ & $6.5 \times 10^{5}$ & 0.63 \\
168 & $4.5 \times 10^{8}$ & $1.5 \times 10^{8}$ & $5.5 \times 10^{5}$ & 0.68 \\
216 & $4.3 \times 10^{7}$ & $1.6 \times 10^{7}$ & $3.0 \times 10^{6}$ & 0.90 \\
312 & $2.1 \times 10^{6}$ & $2.1 \times 10^{6}$ & $5.5 \times 10^{3}$ & 1.07 \\
\hline
\end{tabular}

Total amount of metabolites detected by gas chromatography.

$20 \mathrm{hr}$ designates the sample taken after microbial inoculation of the flask.

TABLE 2.- Microbial counts and gas chromatographically detectable metabolites from NIPER 1 and 6 in tryptic soy broth (TSB)

\begin{tabular}{rcccc}
\hline Time, hr & $\begin{array}{c}\text { NIPER 1 Aerobic, } \\
\text { cfu/mL }\end{array}$ & $\begin{array}{c}\text { NIPER 1 Anaerobic, } \\
\text { cfu/mL }\end{array}$ & $\begin{array}{c}\text { NIPER 6, } \\
\text { cfu/mL } / \mathrm{mL}\end{array}$ & $\begin{array}{c}\text { TGC }{ }^{2} \\
\text { wt\% }\end{array}$ \\
\hline 20 & $1.6 \times 10^{6}$ & $1.3 \times 10^{6}$ & $8.5 \times 10^{5}$ & 0.18 \\
4 & $6.4 \times 10^{5}$ & $1.5 \times 10^{6}$ & $2.3 \times 10^{6}$ & 0.23 \\
8 & $1.0 \times 10^{8}$ & $(3)$ & $1.1 \times 10^{8}$ & 0.24 \\
12 & $6.9 \times 10^{6}$ & $(3)$ & $3.3 \times 10^{8}$ & 0.26 \\
24 & $3.6 \times 10^{10}$ & $3.9 \times 10^{10}$ & $(3)$ & 0.23 \\
48 & $1.8 \times 10^{10}$ & $5.3 \times 10^{9}$ & $3.1 \times 10^{7}$ & 0.24 \\
72 & $1.7 \times 10^{9}$ & $1.6 \times 10^{9}$ & $4.6 \times 10^{8}$ & 0.24 \\
96 & $3.2 \times 10^{9}$ & $2.4 \times 10^{9}$ & $4.9 \times 10^{8}$ & 0.25 \\
120 & $3.9 \times 10^{9}$ & $2.9 \times 10^{9}$ & $2.8 \times 10^{6}$ & 0.24 \\
144 & $1.7 \times 10^{5}$ & $6.2 \times 10^{8}$ & $2.1 \times 10^{6}$ & 0.26 \\
168 & $2.8 \times 10^{8}$ & $3.4 \times 10^{8}$ & $2.3 \times 10^{8}$ & 0.25 \\
216 & $(3)$ & $3.2 \times 10^{8}$ & $3.4 \times 10^{8}$ & 0.25 \\
312 & $5.7 \times 10^{7}$ & $4.2 \times 10^{7}$ & $1.9 \times 10^{5}$ & 0.24 \\
\hline
\end{tabular}

Total amount of metabolites detected by gas chromatography.

$20 \mathrm{hr}$ designates the samiple taken after microbial inoculation of the flask.

${ }^{3}$ Counts were unavailable. 


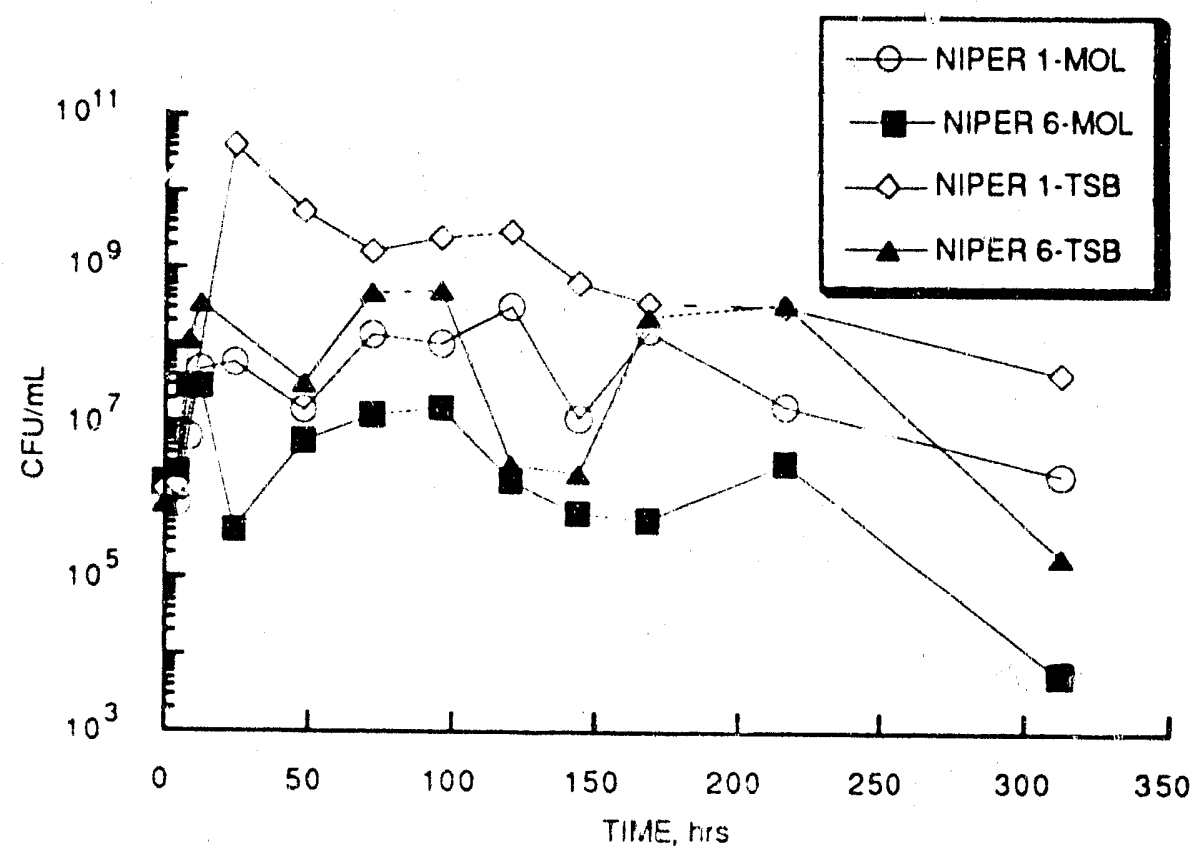

FIGURE 5. Anaerobic microbial counts of NIPER 1 and 6 in TSB and molasses.

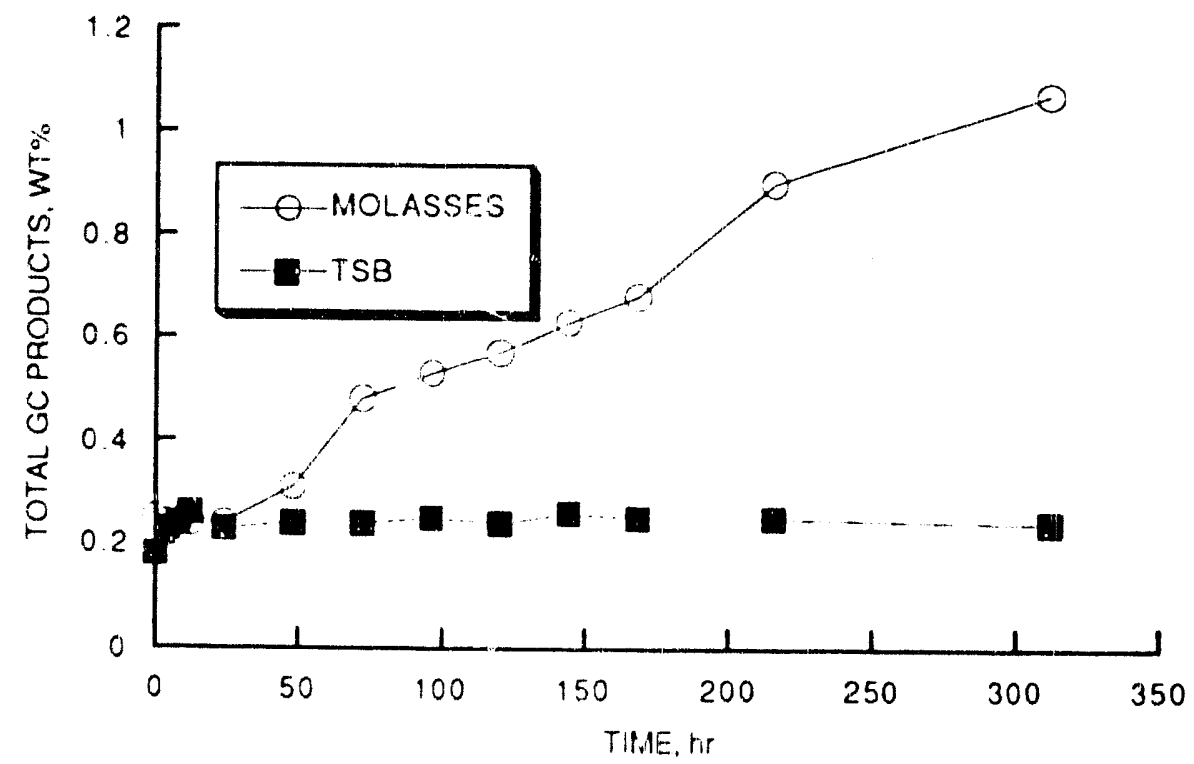

FIGURE 6 - Metabolites detectable by gas chromatography from NIPER 1 and 6 growing in molasses and TSR 
Previous work conducted by NIPER in FY 8.9 showed that use of $1 \%$ sodium bicarbonate was an effective preflush for improved microbial oil recovery efficiency. ${ }^{2}$ it was postulated that perhaps sodium bicarbonate was altering the amounts or types of metabolites produced by NIPER 1 and 6, or perhaps increasing their growth rates. Flask tests and corefloods with $1 \%$ socium bicarbonate and NIPER 1 and 6 showed that there may be a complexity of effects of sodium bicarbonate, both on microbial metabolism and perhaps oil adsorption. To determine the effects of Berea sandstone on microbial metabolism, a second flask tesi was designed to measure the microbial counts, viscosity, and metabolites with sodium bicarbonate, and with Berea sandstone. Flask tests were conducted in the usual manner, with $1 \%$ sodium bicarbonate and approximately 10 grams of Berea sandstone added to the designated flasks. The results are presented in tables 3 and 4 and figures 7 and 8 .

TABLE 3.- Microbial counts and viscosity medsurements from flask tests with Berea sandstone and sodium bicarbonate and NIPER 1 and 6

\begin{tabular}{|c|c|c|c|}
\hline & \multirow[b]{2}{*}{ Viscosity, $\mathrm{CP}$} & \multicolumn{2}{|c|}{ CRUML } \\
\hline & & Aerobic & Anaerobic \\
\hline \multicolumn{4}{|c|}{ IIME $=0$ days $^{1}$} \\
\hline Flask 1 & 1.0 & $1.6 \times 10^{5}$ & $1.7 \times 10^{5}$ \\
\hline Flask 2 & 1.1 & $2.6 \times 10^{5}$ & $4.0 \times 10^{5}$ \\
\hline Flask 3 & 1.0 & $4.8 \times 10^{5}$ & $3.2 \times 10^{5}$ \\
\hline \multirow[t]{2}{*}{ Flask 4} & 1.1 & $4.3 \times 10^{5}$ & $5.0 \times 10^{5}$ \\
\hline & \multicolumn{3}{|c|}{ IIME $=14$ days } \\
\hline Flask 1 & 1.0 & $<1.0 \times 10^{5}$ & $3.2 \times 10^{6}$ \\
\hline Flask 2 & 1.1 & $1.9 \times 10^{7}$ & $2.4 \times 10^{7}$ \\
\hline Flask 3 & 0.9 & $<1.0 \times 10^{5}$ & $1.1 \times 10^{7}$ \\
\hline Flask 4 & 1.1 & $2.4 \times 10^{7}$ & $4.3 \times 10^{7}$ \\
\hline \multicolumn{4}{|c|}{$10 \mathrm{hr}$ designates the sample taken after microbial inoculation of the flask. } \\
\hline \multicolumn{4}{|c|}{ Flask 1 = Control NIPER $1 \& 6$} \\
\hline \multicolumn{4}{|c|}{ Flask $2=1 \%$ sodium bicarbonate added. } \\
\hline \multicolumn{4}{|c|}{ Flask $3=10 \mathrm{~g}$ crushed Berea sanidstone added. } \\
\hline \multicolumn{4}{|c|}{ Flask $4=1 \%$ sodium bicarbonate $+10 \mathrm{~g}$ crushed Berea sandstone added } \\
\hline
\end{tabular}


TABLE 4.- Gas chromatographic products from ilask tests with Berea sandstone and sodium bicarbonate and NIPER 1 and 6

\begin{tabular}{|c|c|c|c|c|}
\hline & Elask 1 & Flask2 & Fask 3 & Flask 4 \\
\hline Product & \multicolumn{4}{|c|}{ Conc, $w \%$} \\
\hline \multicolumn{5}{|c|}{ TIME $=0$ days $^{1}$} \\
\hline Methanol & 0 & 0 & 0 & 0 \\
\hline Ethanol & 0.018 & 0.018 & 0.016 & 0.018 \\
\hline Acetone & $T$ & $T$ & 0 & 0 \\
\hline Isopropyl alcohol & $T$ & $T$ & 0 & 0 \\
\hline Acetic acid & $T$ & $T$ & $T$ & $T$ \\
\hline \multicolumn{5}{|l|}{ Propionic acid/ } \\
\hline n-butyl alcohol & 0 & $T$ & $\mathrm{~T}$ & $T$ \\
\hline Butyric acid & 0 & $?$ & 0.023 & 0 \\
\hline 2,3-Butanediol & 0.058 & 0.456 & 0.065 & 0.056 \\
\hline
\end{tabular}

IIME $=14$ days

\begin{tabular}{lllll} 
Methanol & 0 & 0 & 0 & 0 \\
Ethanol & 0.037 & 0.048 & 0.019 & $T$ \\
Acetone & 0 & 0 & 0 & 0 \\
Isopropyl alcohol & 0 & 0 & 0 & 0 \\
Acetic acid & $T$ & 0.121 & $T$ & 0.362 \\
Propionic acid/ & & & & \\
n-butyl alcohol & 0.026 & 0.01 & 0.025 & 0.011 \\
Butyric acid & 0.173 & 0.365 & 0.336 & 0.343 \\
2.3-Butanediol & $T$ & $T$ & $T$ & 0 \\
\hline
\end{tabular}

10 days designates the sample taken after microbial inoculation of the flask.

$T=$ Trace amount, for all compounds: < 0.01\%; except acetic acid and 2,3-butanediol, which are $<0.05 \%$.

Flask $1=$ Control NIPER $1 \& 6$.

Flask $2=1 \%$ sodium bicarbonate added.

Flask $3=10 \mathrm{~g}$ crushed Berea sandstone added.

Flask $4=1 \%$ sodium bicarbonate $+10 \mathrm{~g}$ srushed Berea sandstone added. 


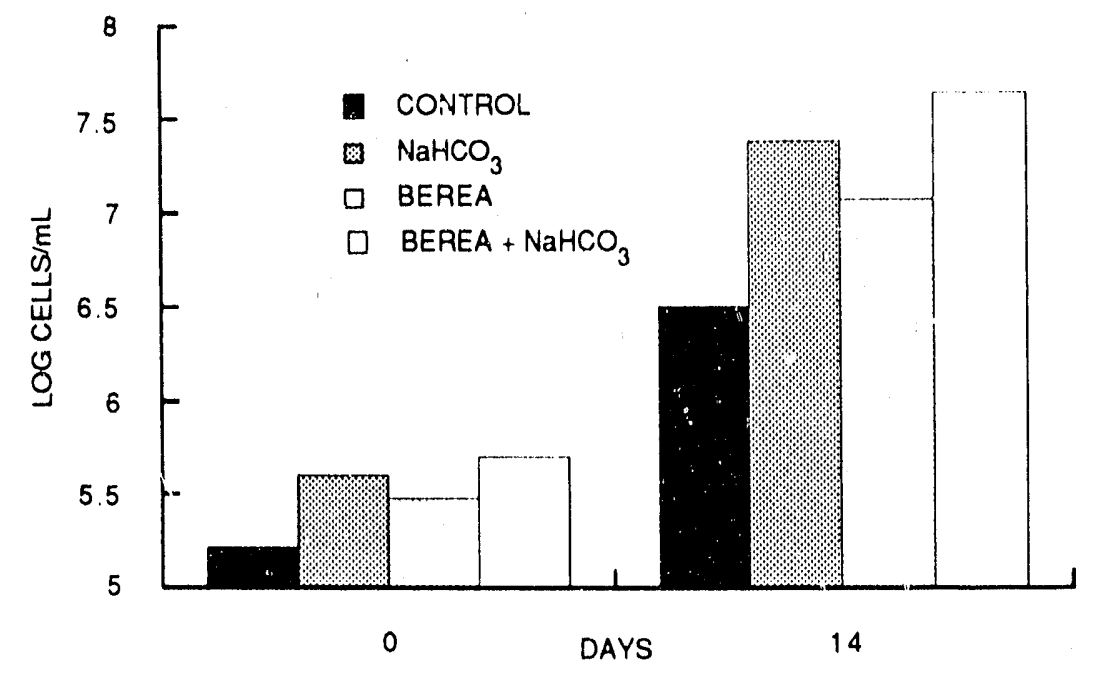

FIGURE 7.- Effect of Berea and $\mathrm{NaHCO}_{3}$ on microbial counts of NIPER 1 and 6.

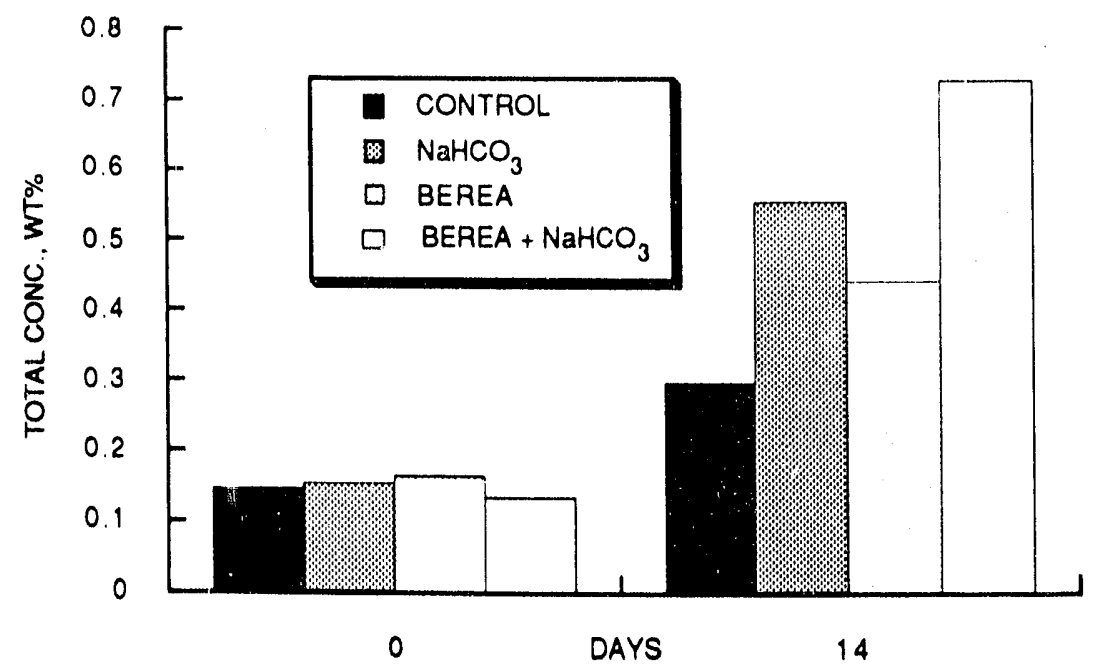

FIGURE 8.- Elfect of Berea and $\mathrm{NaHCO}_{3}$ on total metabolites detected by gas chromatography of NIPER 1 and 6.

The microbial counts, viscositles, and gas chromatographically detectable products from each flask were determined at the initiation of the experiment, and at the end of 2 weeks incubation. The presence of Berea sandstone and sodium bicarbonate does make a significant difference in the amount of certain metabolites produced by NIPER 1 and 6 . In particular, this production of butyric acid and acetic acid is stimulated. Further investigations were conducted measuring the IFTs of these individual chemicals with crude oil; no significant lowering of IFT was achieved. 
Discussions with researchers using the same strain of Bacillus licheniformis have emphasized the differences that nutrients can have on metabolic activity of the microorganisms. An experiment was conducted to ascertain whether NIPER 1 made different metabolites using a modified Medium E nutrient than when using molasses. Interfacial tensions of NIPER 1 in different media using two different hydrocarbons were also measured (table 5). The nutrient medium $E$ is currently used by the University of Texas (Austin) laboratory for their experiments, arid was first described for this microbe by the University of Oklahoma. The filtered molasses as a nutrient stimulated greater metabolite production by our strain of Bacillus licheniformis (figure 9). Iri particular, the production of a tentatively identified butyric acid by NIPER 1 was surprising. Later gas chromatographic analyses indicate that this may be lactic acid. There are twc major differences in these media, one is that modified Medium $E$ is buffered to a pH of 7.0 , while filtered molasses has a pH of around 6.0. Modified Medium E also contained $5 \%$ sodium chloride, which may inhibit our strain of $B$. licheniformis.

TABLE 5.- Interfacial tension (IFT) measurements of NIPER 1 in modified Medium E and molasses containing $5 \% \mathrm{NaCl}$

\begin{tabular}{lll}
\hline & \multicolumn{2}{c}{ IFT, mN/m } \\
\cline { 2 - 2 } Incubation time, hr & Decane & Dodecane \\
\hline
\end{tabular}

MEDIUME

$\begin{array}{rrr}38 & \left({ }^{1}\right) & \left({ }^{1}\right) \\ 64 & \left({ }^{1}\right) & \left({ }^{1}\right) \\ 136 & 4.22 & 5.78 \\ 160 & 6.03 & 2.48 \\ 184 & 7.39 & 4.11\end{array}$

\section{$5 \% \mathrm{NaCl}$ MOLASSES}

38

64

136

160

184
(1)

(1)

6.23

5.33

(1)
(1)

4.68

6.76

(1)

\footnotetext{
Culture produced IFT's greater than could be measured on spinning drop apparatus.
} 


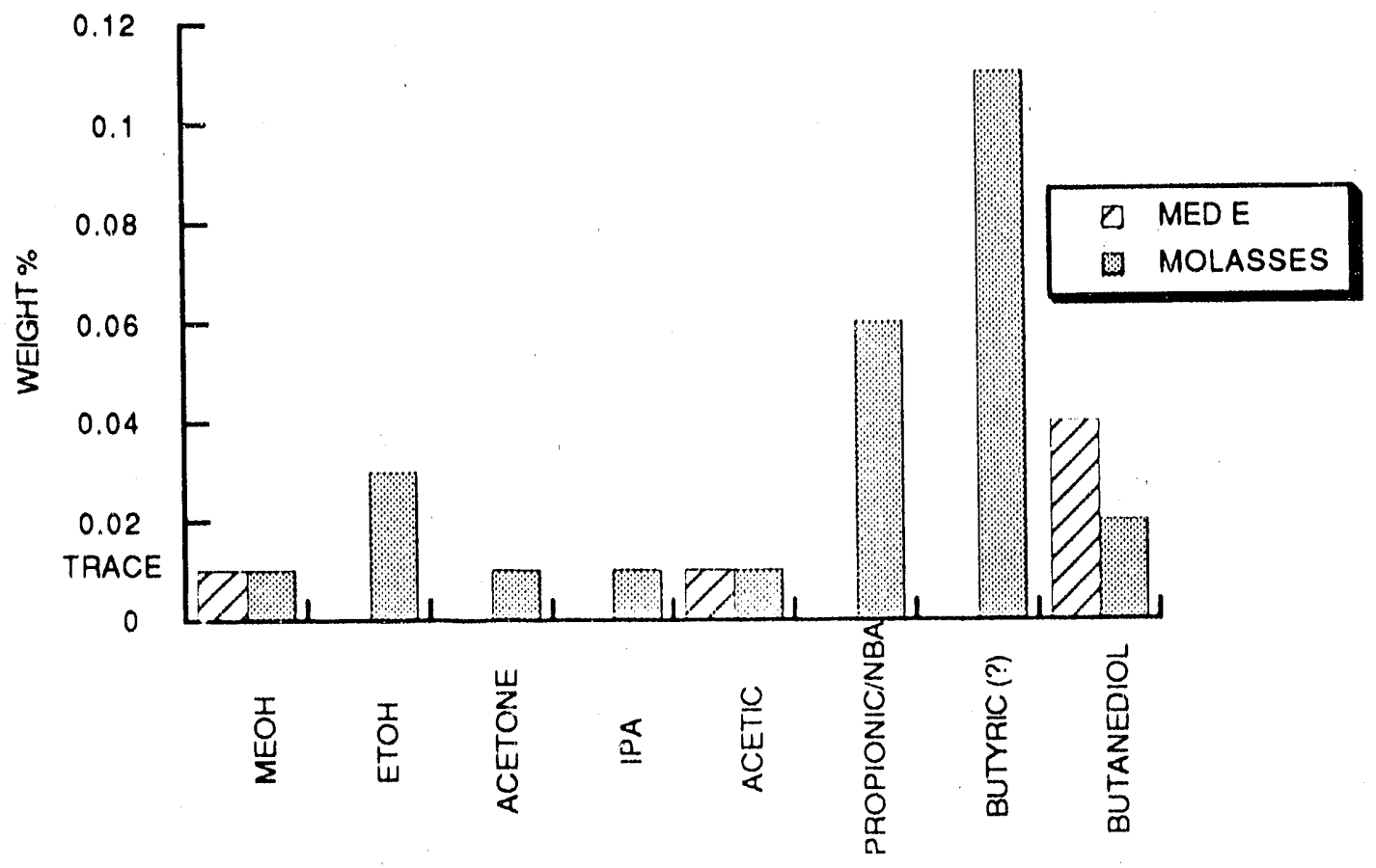

FIGURE 9.- Total metabolites detected by gas chromatography of NIPER 1 grown in Medium E and sterile molasses.

Table 6 and figure 10 show the results of a microbial growth flask test using NIPER 1 and 6 , where the optical density and microbial counts were taken. The counts were surprisingly very low in Medium $E$, particularly for NIPER 6. After about 10 days, the counts appeared to begin increasing, but the lag time for this flask test is much too long for Medium $E$ to be considered a good nutrient for NIPER 1 and 6 . Since this experiment was conducted, Medium E llasks have been inoculated with NIPER 1 and 6 cultures that were adapted to higher salinities, and the results appear to be more favorable. The results from these flask tests emphasize the difference between microbial species and even strains of bacteria. Obviously the strain of Bacillus licheniformis (NIPER 1) that we have been using is different from the one being used at the University of Texas. NIPER 1 has been used in coreflooding experiments at NIPER for more than 5 years. Presumably the microbial strain has continued to adapt during this time and has the capability of transport in porous media, as well as improved oil mobilization properties. ${ }^{2}$ 
TABLE 6.- Microbial counts and optical densities from NIPEF 1 and 6 in modified Medium E

\begin{tabular}{|c|c|c|c|c|}
\hline Time, $\mathrm{hr}$ & $\begin{array}{l}\text { NIPER } 1 \text { Aerobic, } \\
\text { cfu/mL }\end{array}$ & $\begin{array}{l}\text { NIPER } 1 \text { Anaerobic, } \\
\text { cfu/mL }\end{array}$ & $\begin{array}{l}\text { NIPER 6, } \\
\text { cfu/mL }\end{array}$ & $O D_{530^{1}}$ \\
\hline 20 & $6.9 \times 10^{5}$ & $4.1 \times 10^{5}$ & $4.3 \times 10^{3}$ & 0.0 \\
\hline 4 & $4.3 \times 10^{5}$ & $8.0 \times 10^{3}$ & $8.0 \times 10^{2}$ & 0.001 \\
\hline 8 & $9.2 \times 10^{5}$ & $6.0 \times 10^{4}$ & $1.2 \times 10^{4}$ & 0.010 \\
\hline 12 & $1.2 \times 10^{5}$ & $5.0 \times 10^{7}$ & $7.5 \times 10^{4}$ & 0.015 \\
\hline 24 & $1.0 \times 10^{5}$ & $1.5 \times 10^{6}$ & $1.5 \times 10^{6}$ & 0.000 \\
\hline 48 & $4.0 \times 10^{5}$ & $1.0 \times 10^{5}$ & $1.0 \times 10^{4}$ & 0.020 \\
\hline 72. & $2.5 \times 10^{5}$ & $2.5 \times 10^{4}$ & $3.0 \times 10^{4}$ & 0.025 \\
\hline 96 & $9.5 \times 10^{4}$ & $1.7 \times 10^{4}$ & $4.7 \times 10^{3}$ & (3) \\
\hline 120 & $8.0 \times 10^{4}$ & $5.7 \times 10^{4}$ & $3.0 \times 10^{1}$ & (3) \\
\hline 144 & $1.6 \times 10^{5}$ & $3.2 \times 10^{4}$ & $1.4 \times 10^{3}$ & 0.010 \\
\hline 168 & $3.2 \times 10^{5}$ & $7.2 \times 10^{4}$ & $1.5 \times 10^{3}$ & 0.005 \\
\hline 216 & $5.5 \times 10^{4}$ & $3.9 \times 10^{5}$ & $1.4 \times 10^{3}$ & 0.020 \\
\hline 336 & $6.5 \times 10^{7}$ & $2.6 \times 10^{7}$ & $1.2 \times 10^{4}$ & 0.090 \\
\hline 360 & $1.7 \times 10^{7}$ & $8.4 \times 10^{7}$ & $1.0 \times 10^{2}$ & 0.155 \\
\hline 384 & $7.6 \times 10^{6}$ & $2.4 \times 10^{7}$ & (4) & 0.190 \\
\hline 408 & $3.9 \times 10^{7}$ & $3.7 \times 10^{7}$ & $1.2 \times 10^{6}$ & 0.210 \\
\hline 480 & $2.0 \times 10^{4}$ & $4.3 \times 10^{7}$ & $7.7 \times 10^{4}$ & 0.185 \\
\hline 504 & $2.4 \times 10^{4}$ & $6.9 \times 10^{6}$ & $4.7 \times 10^{4}$ & 0.205 \\
\hline
\end{tabular}

Toptical densities measured at 530 nanometers.

2 Time $0 \mathrm{hr}$ designates the sample taken after microbial inoculation of the flask.

${ }^{3}$ Spectrophotometer readings were unreliable.

${ }^{4}$ Counts were unavailable.

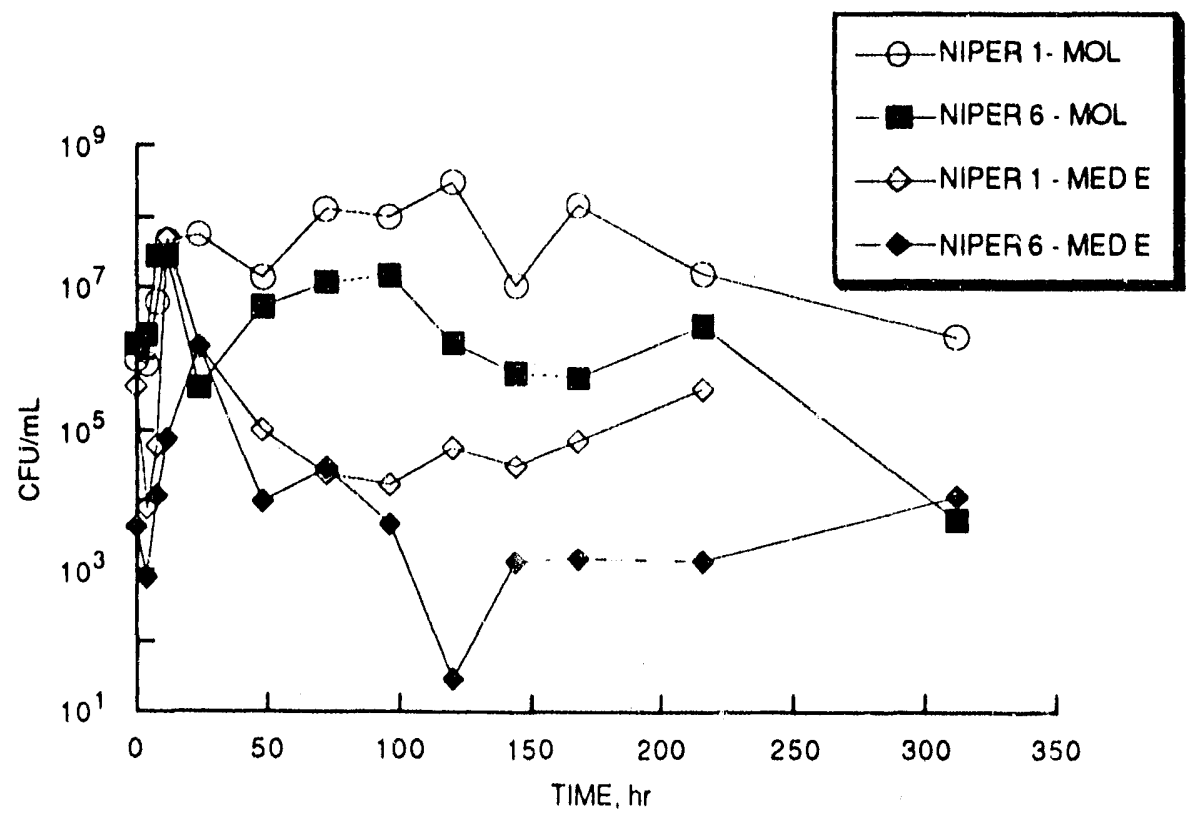

FIGURE 10.- Microbial counts of NIPER 1 and 6 in modified Medium E 
TABLE 7.- IFT determinations between chemicals representing NIPER 1, \& 6 metabolic products and nonane

\begin{tabular}{lcc}
\hline Metabolite & Conc,wt \% & IFT, mN/m \\
\hline Spinning drop tensiometer & & \\
Isopropyl alcohol & 0.05 & 12 \\
Propionic acid & 0.05 & 1.09 \\
Acetic acid & 0.05 & 15.4 \\
Ethanol & 0.05 & 7.5 \\
Butyric acid & 0.05 & 15.2 \\
& & \\
Semi-autemated ring tensiometer & & 48.5 \\
2,3 Butanediol & 0.05 & \\
\hline
\end{tabular}

\section{Interfacial Tension}

Interfacial tension (IFT) measurements were conducted using chemicals known to be metabolic products from the combined microbial cultures NIPER 1 and NIPER 6 formulatinn including: methanol, ethanol, acetic acid, propionic acid, butyric acid, and 2,3-butanediol. Nonane was used as the oil for these experiments, since crude oil from Delaware-Childers field emulsifies very easily and could not be used as effectively with the spinning drop interfacial tensiometer. Nonane was previously determined to have the same equivalent alkane carbon number as that of Delaware-Childers crude oil. Only propionic acid in the nonane system gave an IFT around $1 \mathrm{mN} / \mathrm{m}$ by the spinning drop method (table 7). All other metabolites gave IFTs that were much greater than $1 \mathrm{mN} / \mathrm{m}$. 2,3-butanediol has very little surface activity with nonane and had to be measured using the semi-automated tensiometer (ring detachment) method.

Interfacial tension was also determined using 5-day-old cultures of NIPER 1 and 6 grown anaerobically in unsterile $4 \%$ molassus nutrient containing varying concentrations of sodium chloride. Measurements were made using both the filtered $(0.22 \mu)$ microbial formulation and the unfiltered formulation to determine if there was a difference in IFTs with and without the cells present. Interfacial tensions were also evaluated using two different crude oils. Interfacial tension was slightly lower with crude from the Chelsea-Alluwe field than with oil from Delaware-Childers field. No significant difference was observed in IFT values for the microbial formulation sontaining varying salt concentrations or for unfiltered and filtered microbial formulation--without cells present (table 8). Interfacial tension values were not in the range that would significantly increase capillary number. 
TABLE 8.- Interfacial tension determinations using varying salt concentrations

\begin{tabular}{|c|c|c|c|c|}
\hline \multirow[t]{2}{*}{ 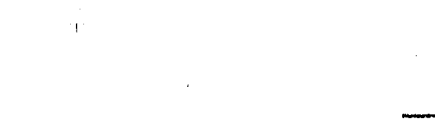 } & \multicolumn{4}{|c|}{ IFT, $\mathrm{mN} / \mathrm{m}$ at varying $\mathrm{NaCl}$ concentrations } \\
\hline & $0 \%$ & $3 \%$ & $5 \%$ & $10 \%$ \\
\hline \multicolumn{5}{|c|}{ Intertacial tension-NIPEB 1 \& 6 anderotic molasses culture (5-days old) } \\
\hline Filtered $1 / 0 C$ oil & 15.5 & 15.3 & 15.3 & 14.9 \\
\hline Unfiltered/DC oil & 13.0 & 14.8 & 15.2 & 14.9 \\
\hline Filtered $1 / C A$ nil & 10.7 & 10.9 & 11.1 & 8.7 \\
\hline Unilteredo a cil & 9.3 & 11.0 & 10.6 & 8.6 \\
\hline \multicolumn{5}{|c|}{ 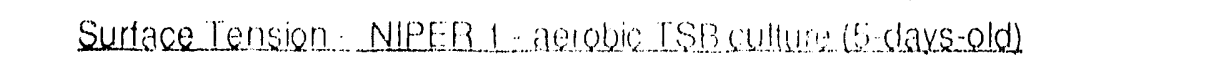 } \\
\hline Unfiltered NIPER 1 in $T S B$ & 383.9 & 43.6 & 55.2 & 56.9 \\
\hline
\end{tabular}

Fillered with 0.22 micron syringe-filler to remove cells.

NIPER 1 was inoculated into tryptic soy broll, (TSB) containing varying concentrations of sodium chloride. After aerobic incubation fur 5 days, surface tension determinations were performed for each unfiltered culture solution. An almost linear increase in surface tension was observed with increased sodium chloride concentration (table 8).

NIPER 1 was also inoculated into filter-sterilized $(0.22 \mu$ pore size) molasses and incubated aerobically at $30^{\circ} \mathrm{C}$. The culture was incubated until a valid reading could be taken using the spinning drop tensiometer. Two highly reined hydrocarbons were used, decane and dodecane, since these had been reported to give low IFT values of $10^{-2} \mathrm{mN} / \mathrm{m}$ with NIPER $1.1^{13}$ The results are shown in table 9. These IFT measurements, although lower than the values oblained with NIPER 1 and 6 , were still not low enough to cause a significant increase in capillary number.

TABLE 9.-- Interfacial tension values of two nutrients containing NIPER 1

\begin{tabular}{lll}
\hline & Med, mN/m \\
\hline & Molasses \\
\hline 136-hour-old culture & & \\
Decane & 4.2 & 6.2 \\
Dodecane & 5.8 & 4.7 \\
Do-hour-old culturt & & \\
Decane & 6.0 & 5.3 \\
Dodecane & 2.5 & 6.8 \\
\hline
\end{tabular}




\section{Micromodel Experiments}

A series of micromodel experiments was performed with I video recorder camera apparatus to document key observations. Previous work in this laboratory has shown that micromodel observations can be correlated with oil recovery data from Berea sandslone coreflooding experiments. ${ }^{11}$ Microbial formulations that recovered a significant amount of crude oil in Berea sandstone cores also mobilized crude oil trapped after waterflooding in micromodels. Figure 11 shows residual oil trapped in the micromodel after waterfloodiny. After microbial injection and incubation, we observed gas droplets that moved into the pore throats containing trapped oil, thereby mobilizing the oil (figs. 12 and 13). Figure 14 shows changes in oil ganglia; elongation and pinching off of many of the oil droplets during waterflooding was observed. These changes indicate lowering of interfacial tension. Some solubilization of the trapped oil droplets was also observed (fig. 15). Several oif droplets began to lighten in color at the edges, producing a type of halo efiect.

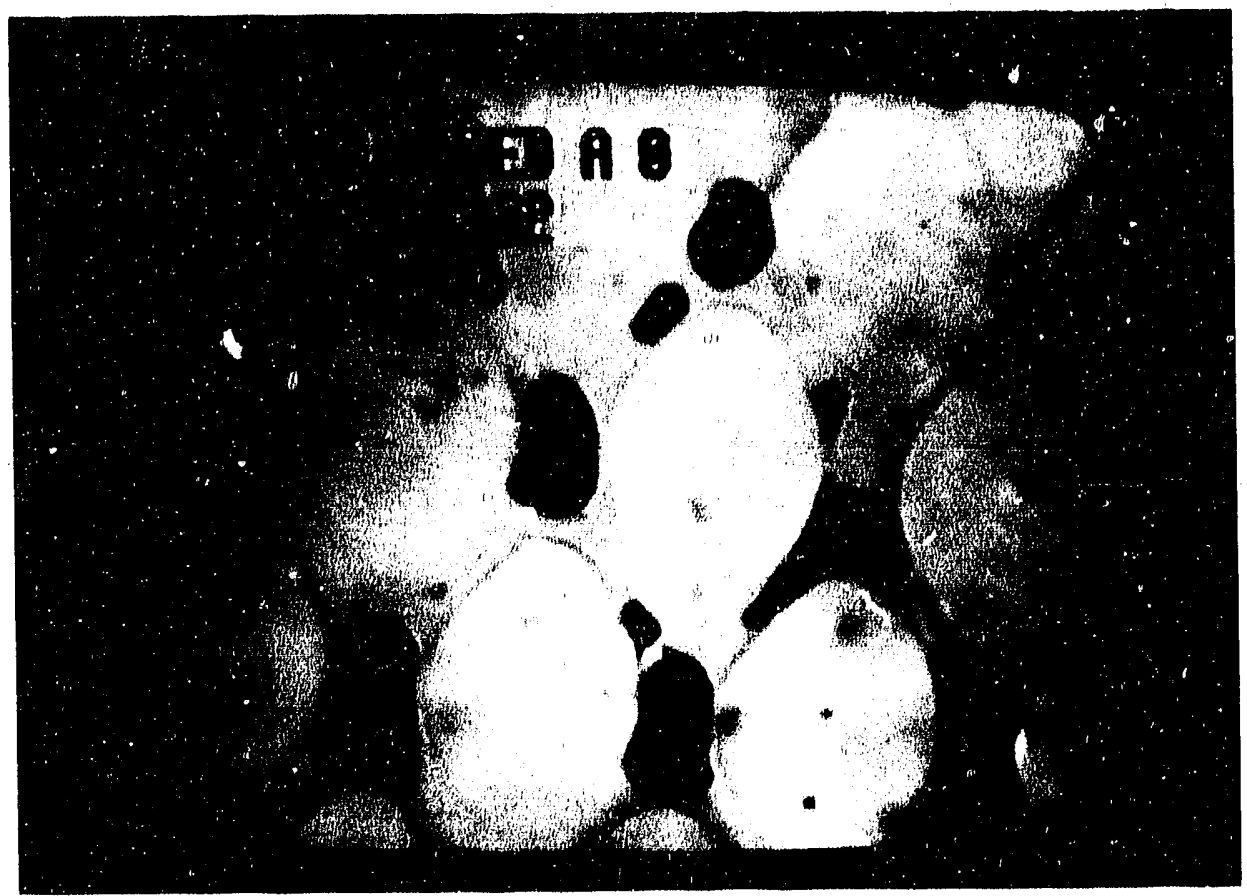

FIGURE11.- Residual crude oil after waterllooding micromodel. 


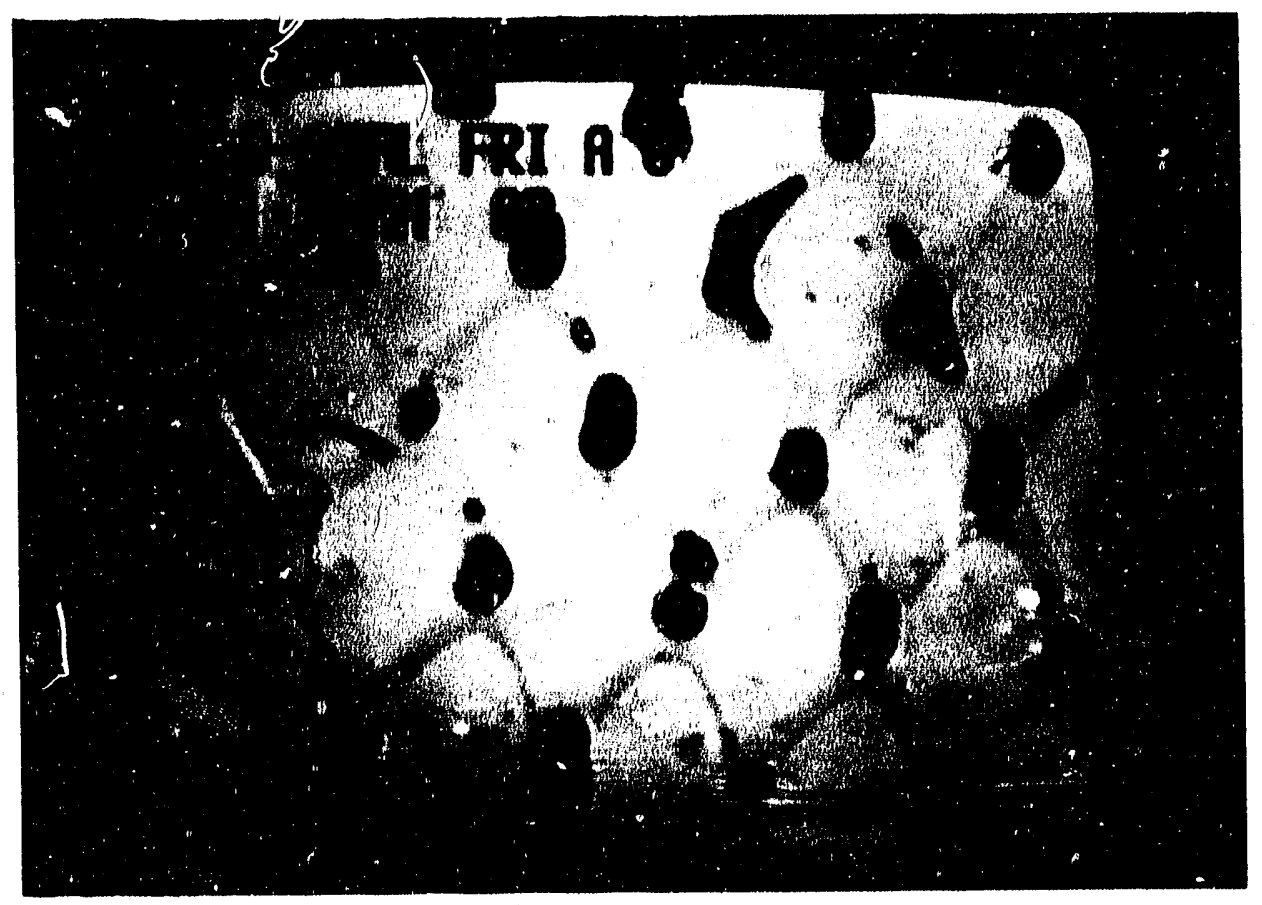

FIGURE 12.- Mlcrobial gas production in micromodel.

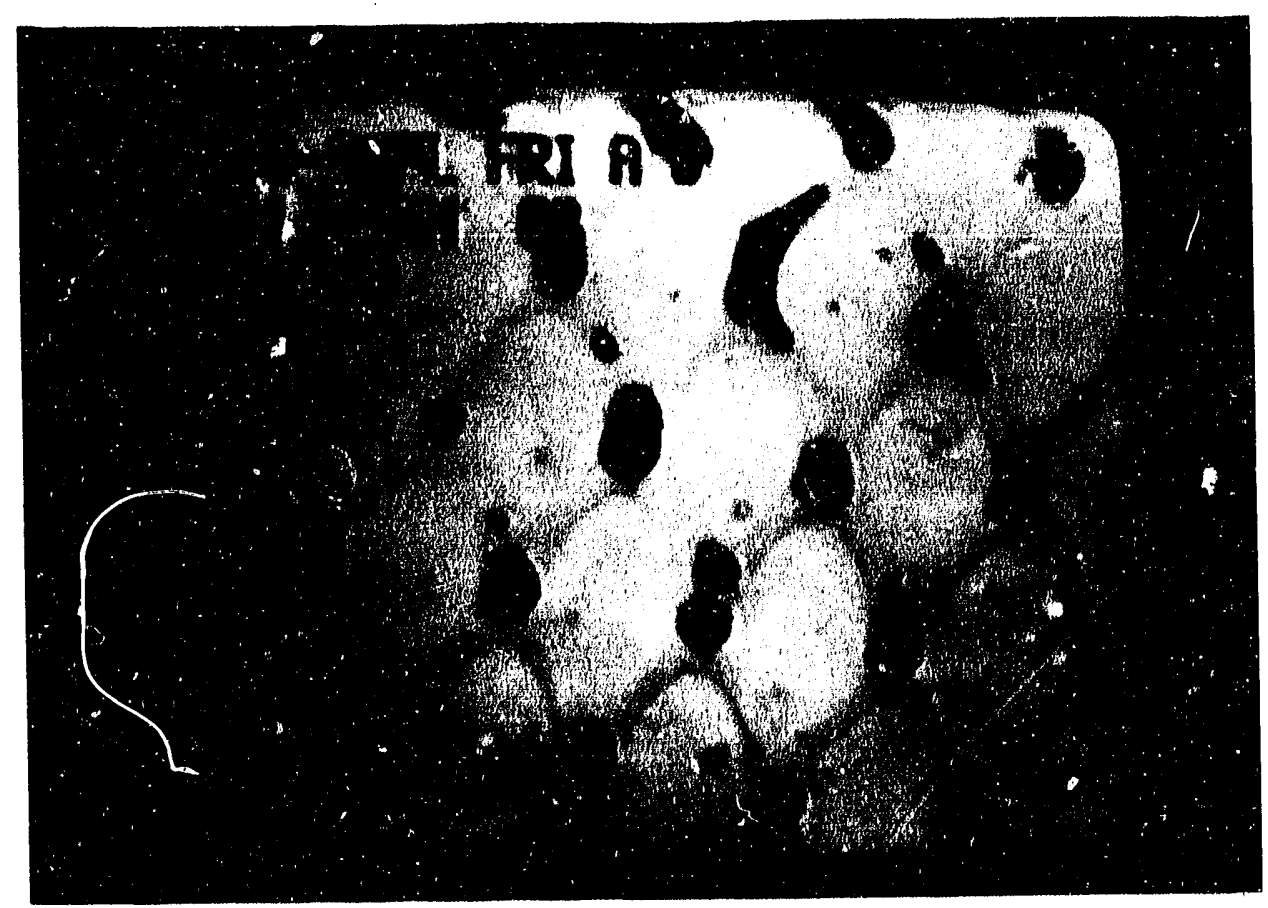

FIGURE 13.- Later view of same location showing gas bulble snapoff. 


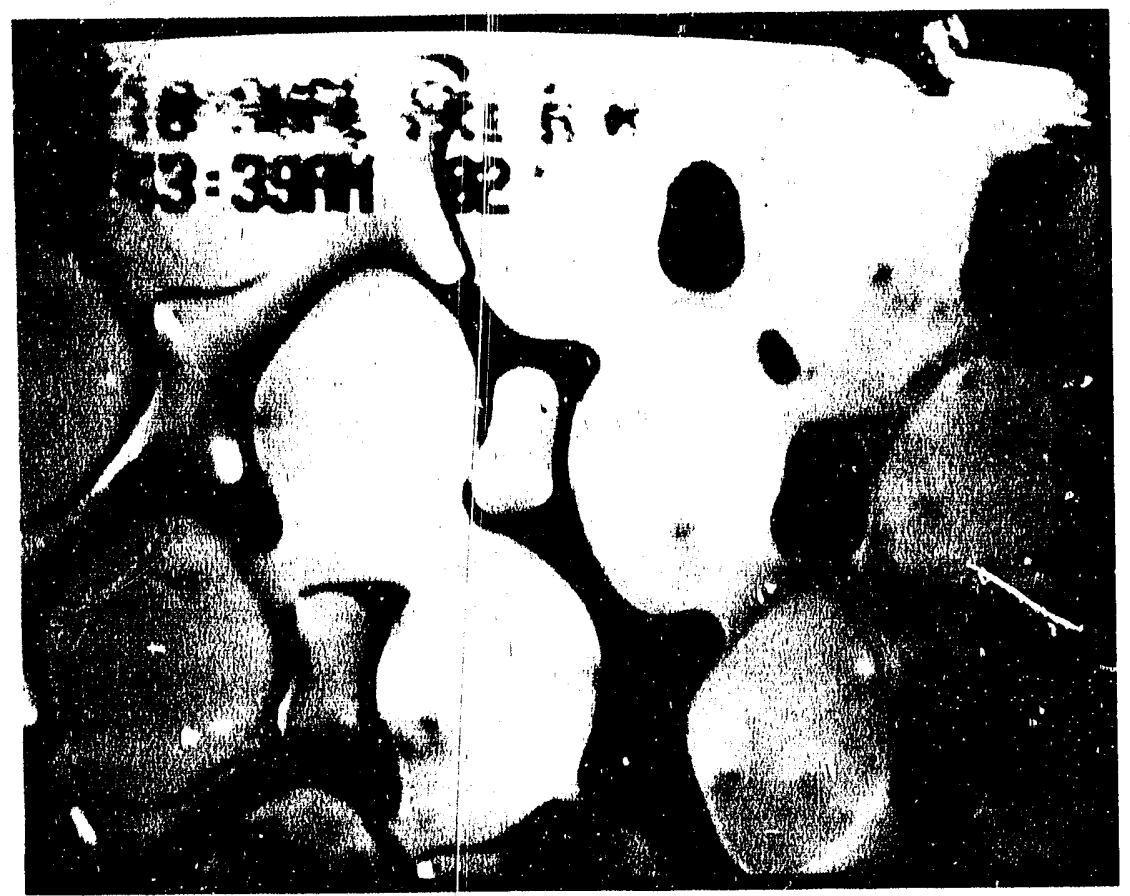

FIGURE 14.- Later view showing oll mobilization from gas production in micromodel.

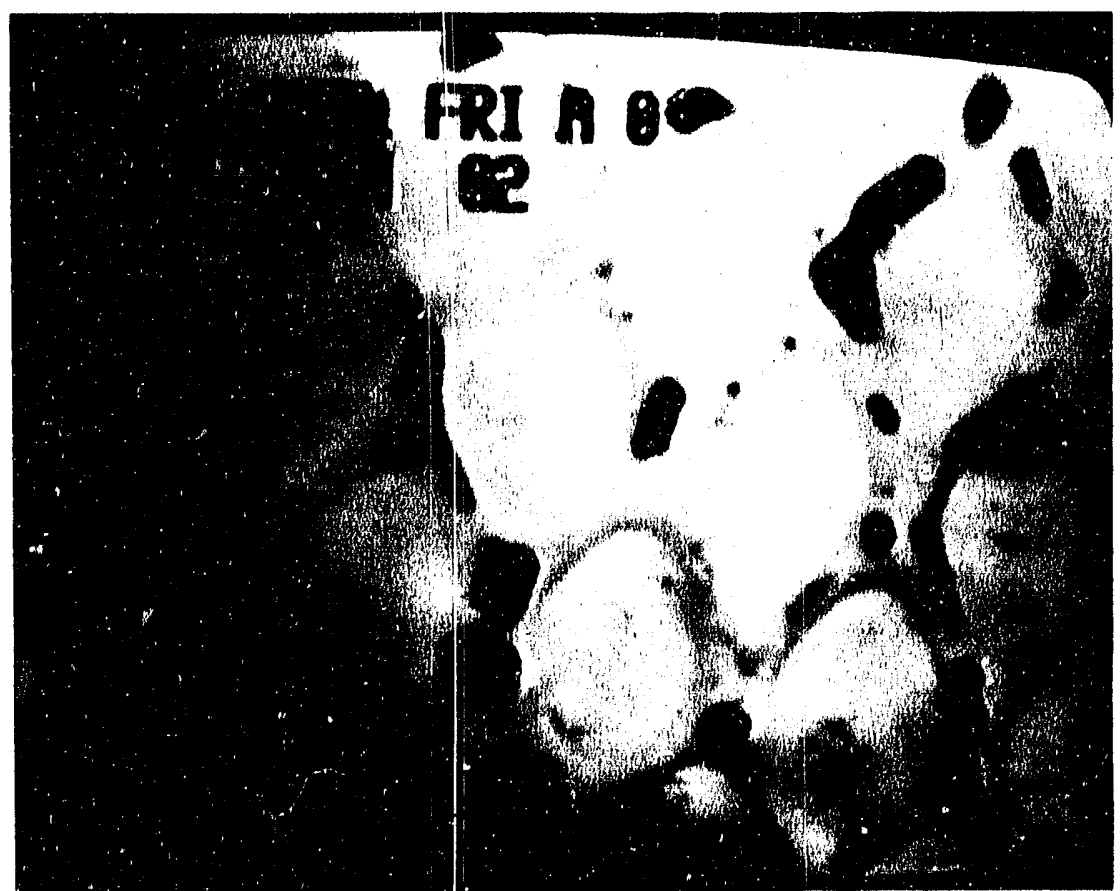

FIGURE 15.- Elongution and mobilization of oil ganglia in micromodel. 


\section{Centrifuge Wettability}

Results of the USBM method centrifuge wettability tests are presented in table 10. NIPER 1 used either alone or in combination with other microbes appears to shift the wettability index significantly in the positive direction toward a more water-wet condition. The core treated with a filtered solution of products (cells removed) appeared to be in the same range of wettability index as the core used with the brine-oil system. The in situ microbial metabolism appears to be involved in wettability alteration since microbial products alorie do not appear to alter wettability.

TABLE 10. - USBM sentrifuge weltabllity tests

\begin{tabular}{|c|c|c|c|c|}
\hline \multicolumn{2}{|c|}{$3 \%$ Sodium chloride brine } & \multicolumn{3}{|c|}{ DC oll and different microbial solutions in TSB } \\
\hline Sample No ${ }^{1}$ & Weltability Index & Sample $\mathrm{No}^{2}$ & Wettability Index & Eormulation \\
\hline $\begin{array}{l}\text { CP66 } \\
\text { CP67 }\end{array}$ & $\begin{array}{l}+0.153 \\
+0.145\end{array}$ & $\begin{array}{l}\text { CP1 } \\
\text { CP2 }\end{array}$ & $\begin{array}{l}+0.315 \\
+0.453\end{array}$ & $\begin{array}{l}\text { Brine/ DC oil } \\
\text { Brine/ DC oil }\end{array}$ \\
\hline CP68 & +0.267 & $\mathrm{CP3}$ & +0.739 & NIPER Bac $1^{3}$ \\
\hline CP69 & +0.196 & CP4 & +0.992 & NIPER Bac 1 \\
\hline $\mathrm{Ci}>70$ & +0.223 & CP23 & +0.927 & NIPER 1 and 3 \\
\hline CP71 & +0.222 & $\begin{array}{l}\text { CP24 } \\
\text { CP9 }\end{array}$ & $\begin{array}{l}+0.763 \\
+0.950\end{array}$ & $\begin{array}{l}\text { NIPER } 1 \text { and } 3 \\
\text { NIPER } 1\end{array}$ \\
\hline \multicolumn{2}{|c|}{ Average $W I=+0.201 \pm 0.046$} & CP10 & +0.959 & NIPER 1 \\
\hline \multirow{2}{*}{\multicolumn{2}{|c|}{$0.5 \%$ Sedium chloride brine }} & CP15 & +0.137 & NIPER 2 \\
\hline & & CP16 & +0.117 & NIPER 2 \\
\hline Sample No ${ }^{1}$ & Wettability Index & CP11 & +0.293 & NIPER 3 \\
\hline CP53 & -0.064 & CP12 & +0.138 & NIPER 3 \\
\hline CP54 & -0.030 & CP13 & +0.283 & NIPER 4 \\
\hline CP55 & -0.082 & CP 14 & +0.262 & NIPER 4 \\
\hline CP56 & +0.063 & CP21 & +0.355 & NIPER 5 \\
\hline & & CP22 & +0.500 & NIPER 5 \\
\hline \multirow{2}{*}{\multicolumn{2}{|c|}{ Average $W 1=-0.028 \pm 0.065$}} & CP19 & +0.310 & NIPER 6 \\
\hline & & CP2O & +0.314 & NIPER 6 \\
\hline
\end{tabular}

Delaware-Childers oil and NIPER 1 \& 6 filtered products in TSB

\begin{tabular}{lc} 
Sample Ne $^{2}$ & Wettability Index \\
\hline CP60 & +0.239 \\
CP61 & +0.117 \\
CP62 & +0.247 \\
CP63 & +0.176 \\
CP64 & +0.237 \\
CP65 & +0.100
\end{tabular}

Average $W I=+0.186 \pm 0.065$

1 The core was aged for 7 days with Delaware-Childers oil at $S_{w i}$.

${ }^{2}$ Core was aged overnight with Delaware-Childers oil.

${ }^{3}$ NIPER Bac 1 is a consortium of 4 microbes used in field test: NIPER 1, NIPER 2, NIPER 3 and NIPER 4.

$W I=$ wettability index 


\section{Amott Wettability}

Results of the Amott wettablity test are presented in table 11. The decrease in relative permeability to the microbial formulation at the ond of the !est shows a decrease in water mobility which would be seen in a trend to more water-wet conditions. The ratio of the permeability to water in the presence of residual oil to the permeability to oil in the presence of connate water (end-point $k_{r w}$ ) is sometimes used as an indicator of wettability-.-a value of less than 0.3 indicales water-wetness and a value near unity indicates oll-wetness ${ }^{13}$. The brine oil core (A1) had a calculated end-point $k_{r w}$ value of 0.355 , whereas the sample tested with microbial formulation (Ad) had a value of 0.220 . The wettability index to water of 0.244 for control core $A 1$ was very close io the USBM centrifuge wettability index values presented in table 4. The wettabllity index to water for the microbial sample was significantly higher; however, this core did imbibe some oil--the wellability index to oil was 0.162 . The difference of 0.422 between the wellability index to the microbial formulation and the wettability index to oll is still twice the value of the wettability index to water for sample A1. The residual oil salumation for sample A4 was also $5.3 \%$ lower, which is consistent with the relative permeability results.

TABLE 11. Summary of Amott wettability lest rosults

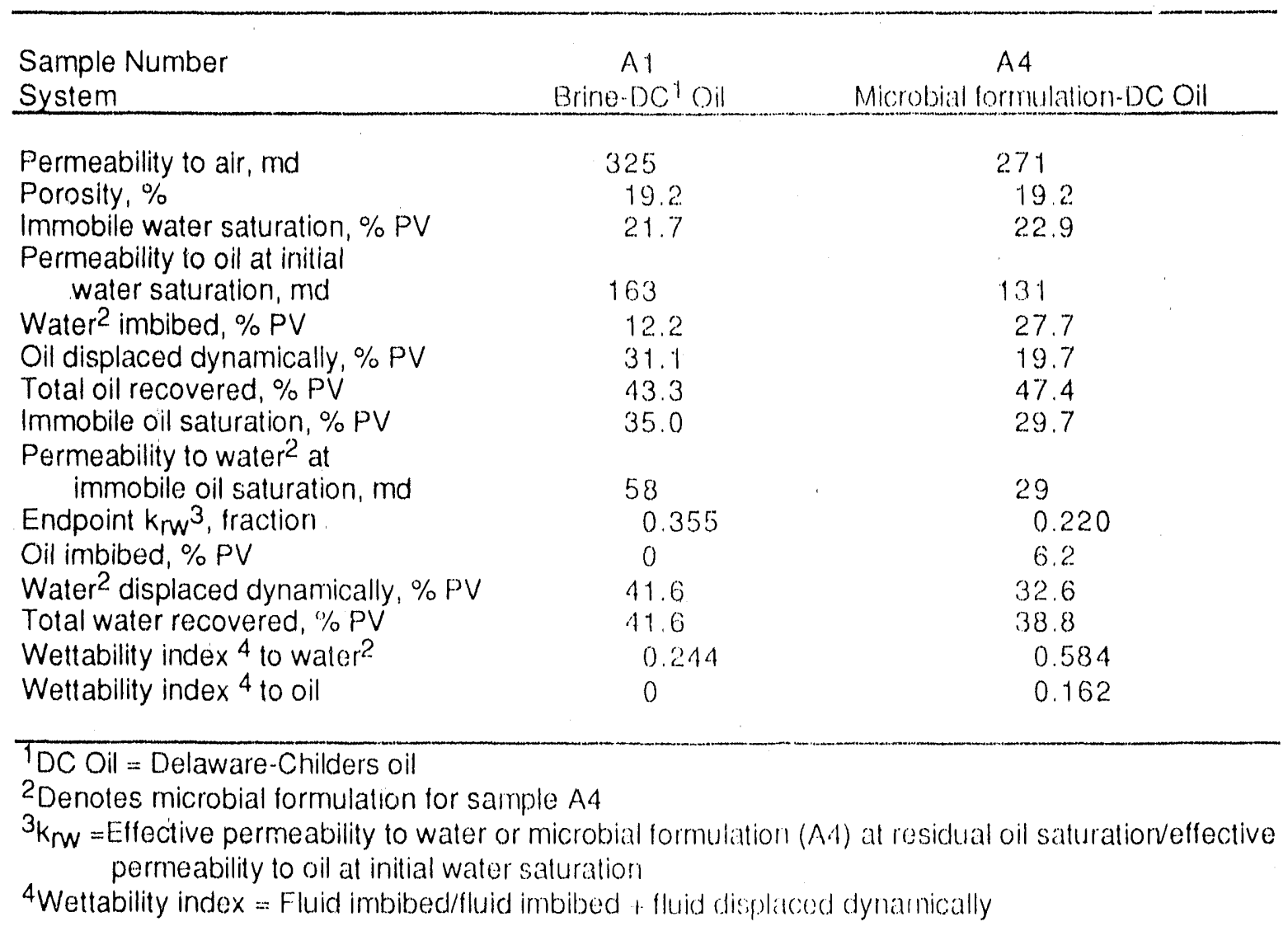




\section{Unsteady-State Relative Permeability Tests}

Relative permeability measurements were conducted on adjacent samples from two sets of Berea sandstone that had different permeability ranges. Each set includes one sample with brine-oil relative permeability and another sample used for microbial formulation-oil relative permeability tests. The two samples from each group are presented together graphically for comparison studies. A summary of relative permeability test results is presented in table 12 . Comparisons of the relative permeability fraction curves and ratio curves are presented in figures 16 and 17, respectively. A higher relative permeability to oil is apparent in both microbially flooded samples as compared to brine-flooded samples. The higher permeability core exhibited the effect much earlier in the flood. A slight shift to the right can be observed in the ratio curves of microbially flooded samples, which indicates that sample $R 8$ had an increase in oil production as compared with the brine sample toward the latter half of the flood. Sample R2 exhibited a signiticant decrease in relative permeability to water throughout the flood. This higher permeability core, flooded with a microbial formulation that had been incubated 24 hours, exhibited an increase in oil productior and relative permeability to oil immediately and continuing throughout the flood.

Oil recovery before water breakthrough was derived irom the fractional flow equations using the viscosity of the Delaware-Childers oil. The samples wateriboded with the microbial formulation had a higher oil recovery before water breakthrough. A greater difference was observed in the relative perrieability characteristics for the higher permeability sample that had been flooded with the 24-hour microbial culture

TABLE 12.- Summary of unsteady-state relative permeability tests

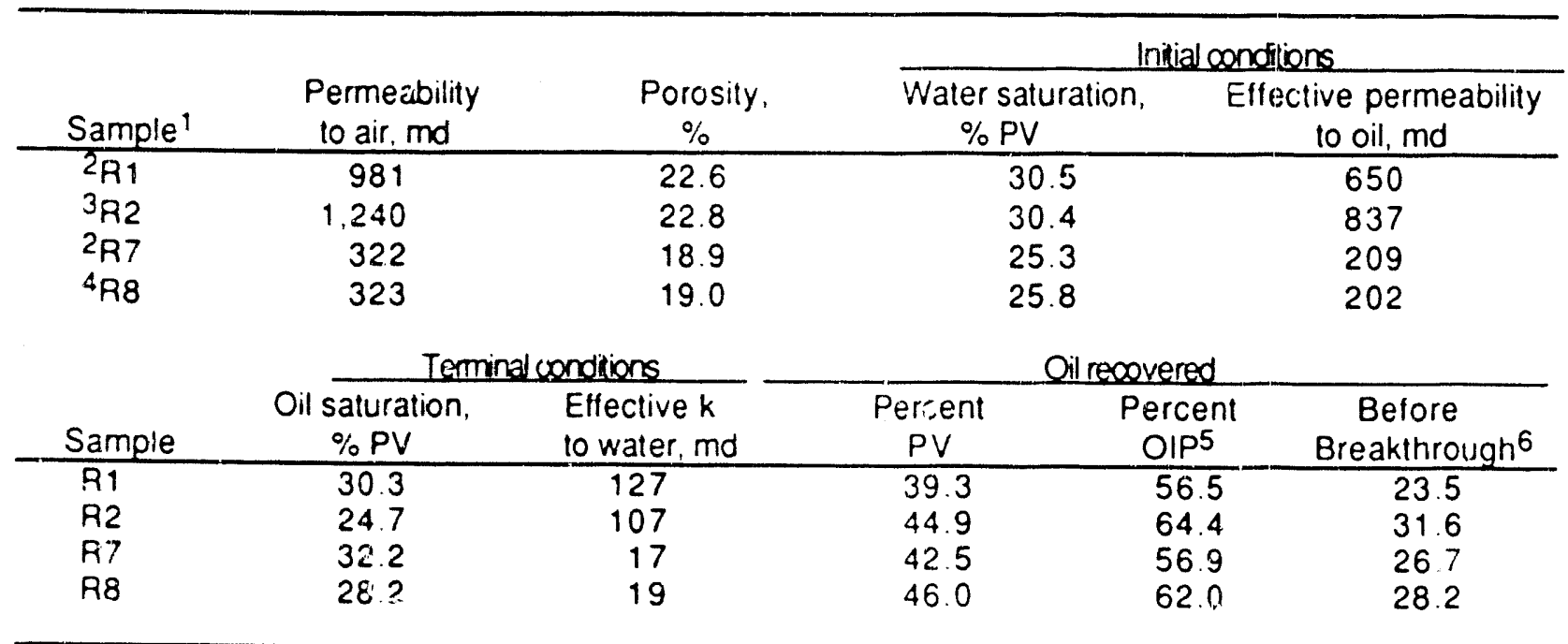

\footnotetext{
All cores were aged for 1 week with Delaware-Childers oil.

${ }^{2}$ Brine/oil (mineral oil) relative permeability.

324 -hour micrisbial solutionoil (mineral oil) relative permeability.

45-cay old microbial solution (optimal biosurfactant production)/oil (mineral oil) relative permeability.

${ }^{5} \mathrm{OIP}=$ Oil in place

${ }^{6}$ Calculated using viscosity of Delaware-Childers oil.
} 

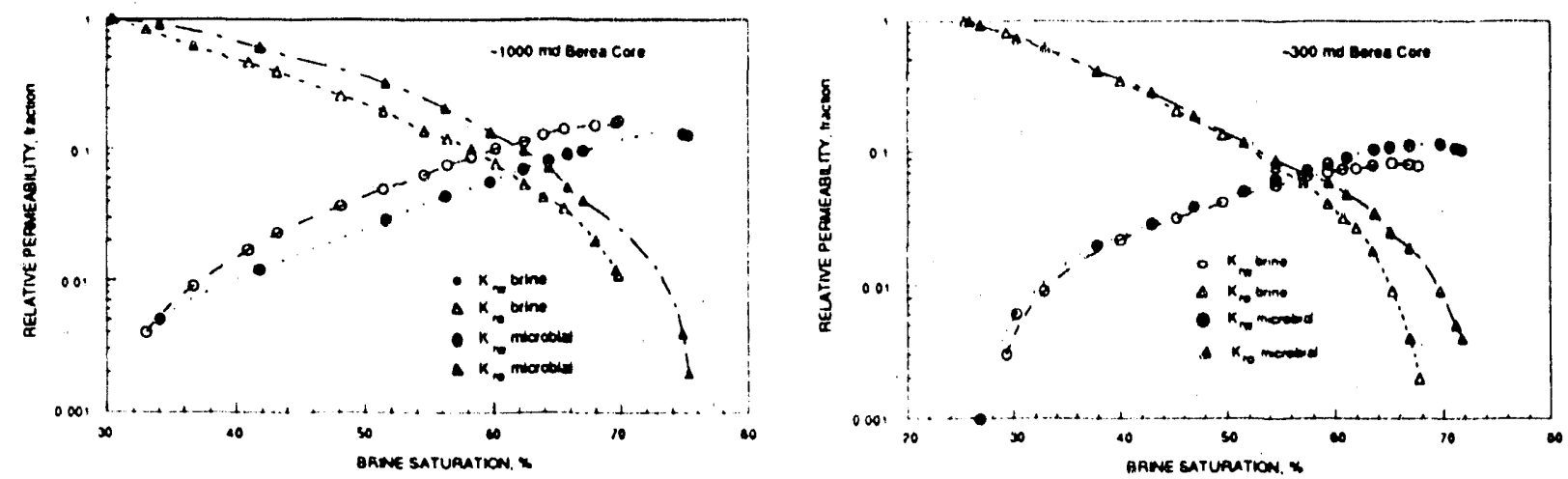

FIGURE 16.- Unsteady-state relative permeability fraction surves for cores with different permeability values.
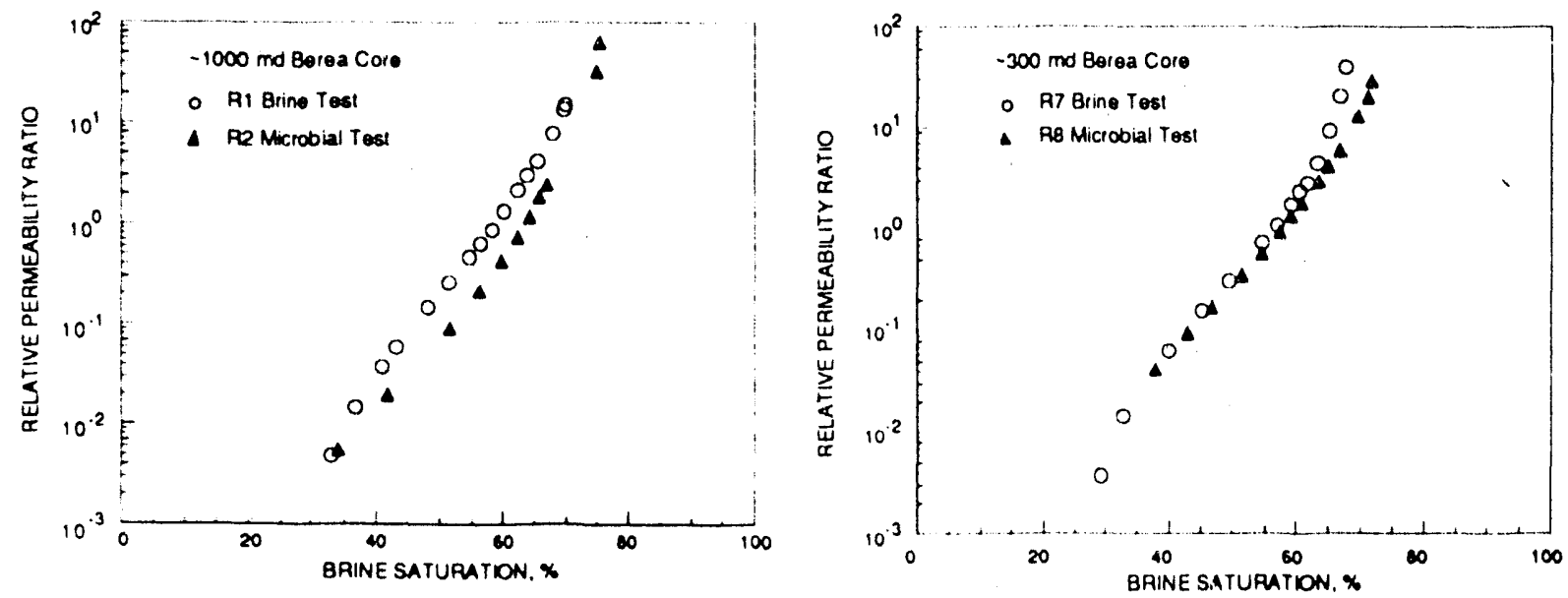

FIGURE 17.- Unsteady-state relative permeability ratio curves for cores with different permeability values

\section{CONCLUSIONS}

Data from several different flask tests have shown that by altering the nutrients and/or some of the environmental conditions, the metabolites produced by NIPER 1 and 6 can be changed. No one metabolite detectable by gas chromatography has been shown to significantly alter the IFT. The biosurfactants produced by NIPER 1 and 6 are probably the most important metabolites for oil mobilization, although even the metabolites themselves cannot significantly mobilize crude oil when the cells are riot present. Flask experiments comparing microbial growth and metabolite production in molasses, molasses with sodium bicarbonate and/or Berea sandstone, and modified Medium $E$ have demonstrated a great variety of microbial growth patterns and metabolite production. Although the interfacial tensions measured by the microbial cells with various crude oils and hydrocarbons are not in the range usually responsible for oil mobilization, there may be more of a variation in IFT behavior than can be measured at the present time. As an exaniple, one of the keys to improved oil mobilization by NIPER 1 
and 6 appears to be the presence of the metabolically active cells. Because the localized transient concentration of surfactant production cannot be measured at the oil/water interface, likewise, the IFTs at this intertace may be transient and much lower than the overall values obtained.

Several biochemical assays were examined for their ability to detect and quantitate the biosurfactants produced by NIPER 1 and NIPER 6 using a spectrophotometric method. The biosurfactant from NIPER 1 is reported to have a peptide and a lipid moiety which can be detected using thin-layer chromatography; however, to quantitate the amount of surfactant, a colorimetric assay would be the easiest and most effective method to provide a standard concentration curve. Al this time, there is no rapid or easy method to quantitate the NIPER 1 and 6 biosurtactants.

Interfacial values determined using the spinning drop apparatus were not in the range to cause an appreciable increase in capillary number. However, results from the micromodel studies show that interfacial tension must have been lowered by the microbial formulation in order to effectively mobilize the crude oil. Gas production by the microbial formulation was also responsible for some oil mobilization; however, in previous micromodel studies we have shown that a microbial formulation that produces only gas does not mobilize a significant amount of crude oil. The microbial surfactant production is important for crude oil mobilization.

Studies are continuing to isolate and quantitate the biosurfactant produced by NIPER 1. Sarkar, et al. ${ }^{14}$ are reporting values in the range of $0.03 \mathrm{mN} / \mathrm{M}$ for Bacillus licheniformis JF-2. At this point, we have not been able to get values in this range. One of the major differences appears to be the nutritional requirements for NIPER 1 versus the Bacillus licheniformis JF-2 strain obtained by the University of Texas, and recent reports from Idaho National Engineering Laboratory. ${ }^{15}$ These nutritional differences do not appear to be related to oil mobilization, however, since we are able to achieve much better oil recovery efficiencies with the NIPER strain.

Microbial formulations containing NIPER 1 appear to shift the wettability index of Berea core samples from jusi slightly water-wet to a more positive value. The microbial cells are involved in this wettai lity alteration since no change was observed in samples tested with filtered microbial products (cells removed). In situ microbial metabolism appears to be involved in wettability alteration. Results of the Amott wettability test indicate that the wettability index 10 water for the microbially treated core was twice that of the value for the control core, indicating a more water-wet condition.

A significant decrease was seen in $k_{r w}$ curves ( a decrease in water mobility) for (wo of three samples containing microbial formulation. Both relative permeability test samples showed an increase in relative permeability to oil; although, one sample containing microbial formulation had a $20 \%$ higher initial effective permeability to oil. However, it has been shown that cores with varying permeabilities but the same rock type (pore geometry) have almost identical relative permeability characteristics. ${ }^{16}$ A slight shift to the right was observed at the brine saturation at which oil and water relative permeabilities are equal (crossover). Samples tested with the microbial formulation had higher oil recovery before water 
breakthrough and lower residual oil saturations. Steady-state water-oil relative permeability tests may provide more meaningful data since Delaware-Childers crude oil, which has a low viscosity of $7.5 \mathrm{cP}$, could be used instead of refined mineral oil.

Although all wettability tests with microbial formulations exhibited a change in the direction of a more water-wet condition, changes in the range of neutral wettability are very hard to distinguish. Berea sandstone is very water-wet, and any alteration :oward a more oil-wet condition is difficult to attain. Tests performed on a core that is definitel in the range of oil-wet would be helpful in further evaluating the effects of microbial systems on wettability.

\section{REFERENCES}

1. Lake, L. W. Enhanced Oil Recovery. Prentice Haill, Englewood Cliffs, New Jersey, 1989.

2. Bryant, R. S., K. L. Chase, K. M. Bertus, and A. K. Stepp. Effects of Chemical Additives on Microbial Enhanced Oil Recovery Processes. Dept. Of Energy Report No. NIPER-442, December, 1989.

3. Amott, E. Observations Relating to the Wettability of Porous Rock, Trank., AIME 216, pp 156-62, Technical Paper No. 8069, 1959.

4. Donaldson, E. C. Equipment and Procedures for Fluid Flow and Wettability Tests of Geological Materials, Dept. of Energy Report No. DOE/BETC/IC 79/5, May, 1980.

5. Bryant, R. S., T. E. Burchfield, D. M. Dennis, and D. O. Hitzman. Microbial-Eninanced Waterflooding,: Mink Unit Project. SPE/DOE paper 17341, SPE Reservoir Engineering , Vol. 5, No. 1, February, 1990.

6. Jenneman, G. E., M. J. Mclnerney, and R. M. Knapp. Microbial Penetration through NutrientSaturated Berea Sandstone. Applied and Environmental Microbiology, Aug. 1985, pp. 383-391.

7. American Public Health Association, \#907. Standard Plate Count, In: Standard Methods for the Examination of Water and Wastewater. 15th edition, 1980.

8. Standard Test Method for Interfacial Tension of Oil Against Water by the Ring Method. ASTM Designation: D971-82.

9. Wade, W. H., R. S. Schecter, and J. L. Cayias The Measurement of Low Interfacial Tension via the Spinning Drop Technique, Adsorption at Intertaces, ACS Symposium Series, No. 8, 1975.

10. Chatzis, I. Photofabrication Technique of 2-D Micromodels. PPRC Report No. 82-12, New Mexico Petroleum Recovery Research Center, March 1, 1982.

11. Bryant, R. S. arid J. Douglas. Evaluation of Microbial Systems in Porous Media for EOR. SPE Reservoir Engineering, Vol. 3, No. 2, May, 1988. SPE Paper No. 16284.

12. Johnson, E. F., D. P. Bossler, and V. O. Naumann. Calculation of Relative Permeability from Displacement Experiments. Trans, AIME 216, pp. 370-372, January, 1959.

13. Raza, S. H., L. E. Treiber, and D. L. Archer. Wettability of Reservoir Rocks and its Evaluation. Producers Monthly, Vol. 32, No. 4, April, 1968. 
14. Sarkar, A. K. , M. M. Sharma and G. Georgiou. Compositional Numerical Simulation of MEOR Processes. Pres. at the 1990 Conference on Microbially Enhanced Oil Recovery, Norman, Okla., 1990.

15. Thomas, C. E. MEOR Research on Heavy Oils. Pres. at the 1990 Conference on Microbially Enhanced Oil Recovery, Norman, Okla., 1990.

16. Morgan, J. T. and D. T. Gordon. Influence of Pore Geometry on Water-Oil Relative Permeability. J. Pet. Tech., October, pp.1199-1208, 1970. 

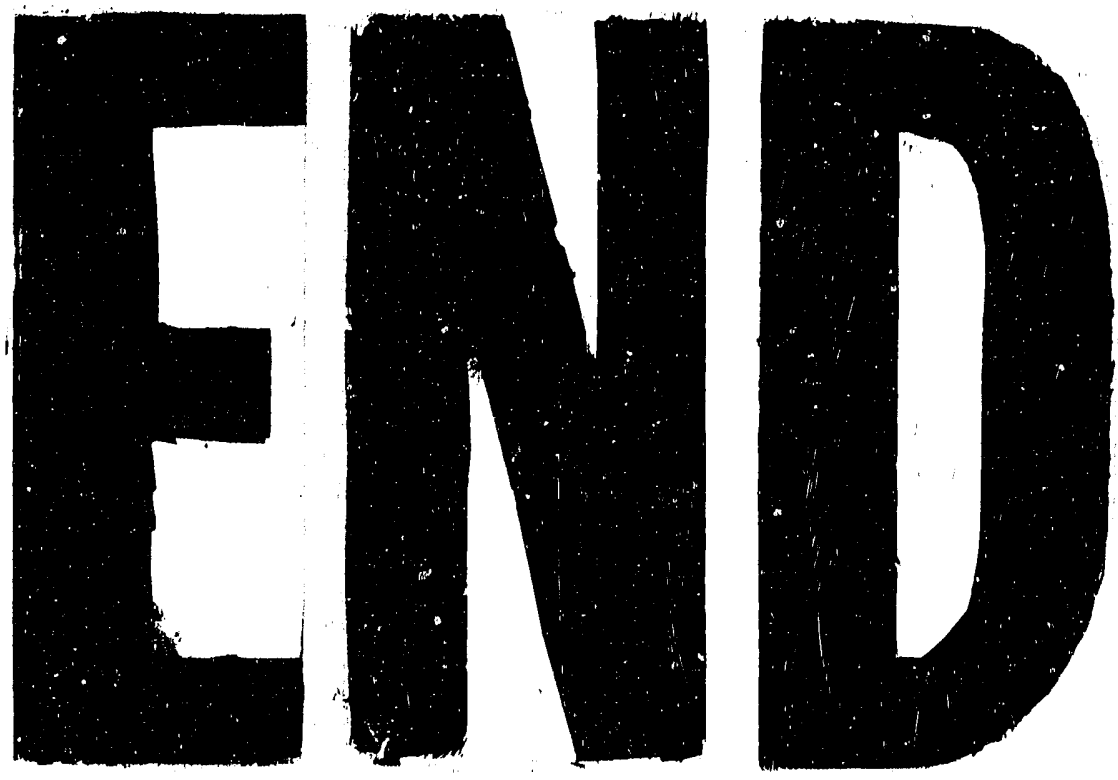

4
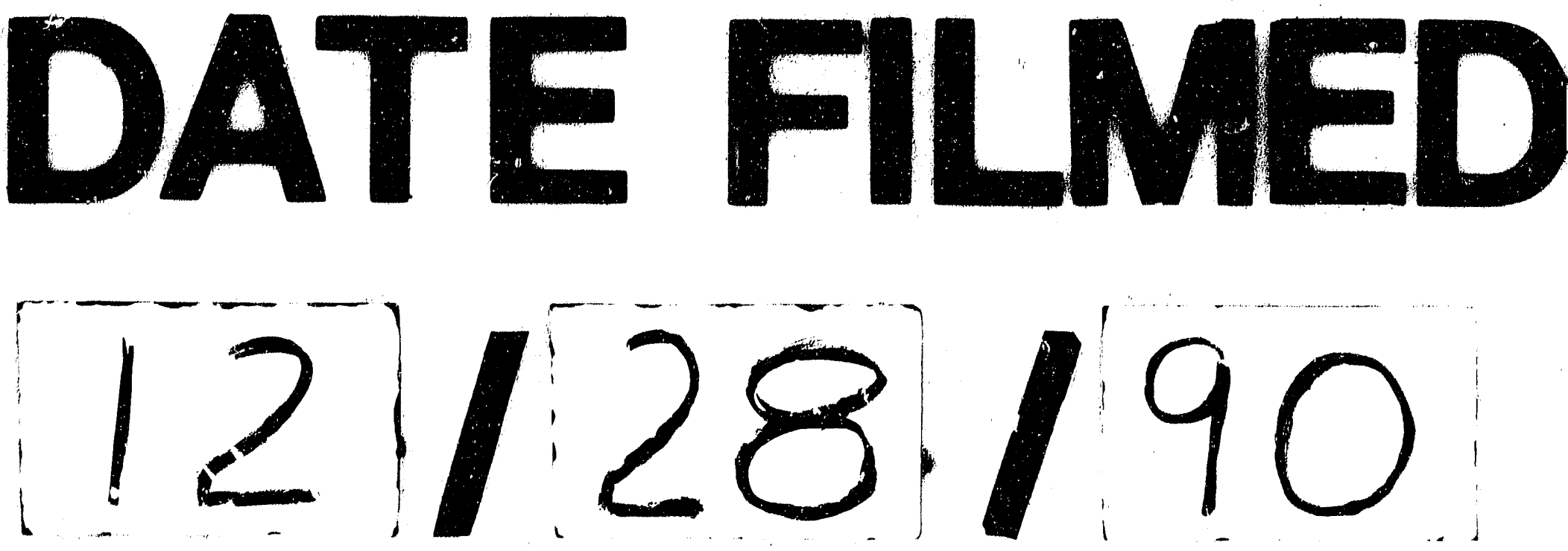
